\title{
Estimates for multiple stochastic integrals and stochastic Hamilton-Jacobi equations
}

\author{
Vassili N. Kolokol'tsov, René L. Schilling and Alexei E. Tyukov
}

\begin{abstract}
We study stochastic Hamilton-Jacobi-Bellman equations and the corresponding Hamiltonian systems driven by jump-type Lévy processes. The main objective of the present paper is to show existence, uniqueness and a (locally in time) diffeomorphism property of the solution: the solution trajectory of the system is a diffeomorphism as a function of the initial momentum. This result enables us to implement a stochastic version of the classical method of characteristics for the Hamilton-Jacobi equations. An -in itself interesting- auxiliary result are pointwise a.s. estimates for iterated stochastic integrals driven by a vector of not necessarily independent jump-type semimartingales.
\end{abstract}

\section{Introduction}

Over the last few years interest in stochastic Hamilton-Jacobi-Bellman (HJB for short) equations has increased, see e.g. the papers [R], [So], [DaPDe] and references given there. The HJB equations are important as they describe the evolution of optimally controlled systems with random dynamics, but they are also useful tools when studying various classes of stochastic models in probability theory and mathematical physics. Presently, the notion of stochastic HJB equation is used in two different contexts: firstly, for classical differential equations with a random Hamiltonian and, secondly, for truly stochastic differential equations where the Hamiltonian includes a non-homogeneous semimartingale term which does not allow to write down the corresponding equation in classical form.

2000 Mathematics Subject Classification: Primary: 70H20, 60H15, 60H05. Secondary: 60G51, 60J75, 70H05.

Keywords: stochastic Hamilton-Jacobi equation, Hamiltonian system, method of stochastic characteristics, iterated stochastic integral, semimartingale, Lévy process. 
In the sequel we will consider the second type of HJB equations, that is to say equations of the form

$$
d S+H\left(x, \frac{\partial S}{\partial x}\right) d t+c(x) d \xi_{t}=0, \quad x \in \mathbb{R}^{d}, \quad t>0,
$$

where $H: \mathbb{R}^{2 d} \rightarrow \mathbb{R}$ and $c: \mathbb{R}^{d} \rightarrow \mathbb{R}^{d}$ are smooth functions and $\xi_{t}$ is a stochastic process (driving noise) in $\mathbb{R}^{d}$. The corresponding Hamilton system is then

$$
\left\{\begin{array}{l}
d x=\frac{\partial H}{\partial p} d t \\
d p=-\frac{\partial H}{\partial x} d t-\frac{\partial c}{\partial x} d \xi_{t} .
\end{array}\right.
$$

The equation (1.1) driven by a Wiener process $\left\{\xi_{t}\right\}_{t \geqslant 0}$ was considered in [K1], [K2], and [TrZ1], [TrZ2] for various classes of real $H$ and $c$. The corresponding case of complex valued $H$ and $c$ was taken up in [K3]. The main objective of the present paper is to study the equations (1.1), (1.2), where $\left\{\xi_{t}\right\}_{t \geqslant 0}$ is a Lévy noise without Brownian part and to develop a stochastic analogue of the theory of classical (i.e. smooth in $x$ ) solutions of the Cauchy problem for equation (1.1). Generalised solutions can then be constructed (see $[\mathrm{KT} 2]$ ) in the same way as they are constructed for the case of a Wiener process $\left\{\xi_{t}\right\}_{t \geqslant 0}$ in [K1], [K2] (see also [KMa]).

For this programme we need to be able to solve a boundary value problem for the stochastic Hamilton system (1.2). The analysis of the latter problem is the second main topic of our paper. Boundary value problems for Hamilton systems of type (1.2) with a Wiener process $\left\{\xi_{t}\right\}_{t \geqslant 0}$ and their connections with the calculus of variations were investigated in [K2]. However, the proof of the existence and uniqueness of the solution of the boundary value problem was only sketched in [K2]. In this paper we give complete proofs of the corresponding results for Hamilton systems driven by Lévy noise without a Brownian part.

An important tool for the analysis of the behaviour of the solutions for Hamilton systems is the study of their linearised approximations (equation in variations). These linearised approximations turn out to be linear nonhomogeneous Hamilton systems. Using perturbation theory we can derive a representation of the solutions of such linear systems as series of multiple stochastic integrals. In order to prove the convergence of these series, we are led to the third topic of the article - obtaining estimates for multiple stochastic integrals. In the present paper we use these estimates as auxiliary tools for the study of linear stochastic Hamilton systems. We believe, however, that they are of independent value. Let us mention here the paper [Ta], where a rather general linear system driven by Brownian motion 
was considered, convergence of the series from perturbations theory proved, and necessary estimates for multiple integrals obtained. Multiple stochastic integrals with respect to general semimartingales or infinitely divisible processes were also considered, see e.g. [KwW], [Sz] and references given there.

Let us give a brief outline how our paper is organized. In Section 3 we obtain estimates for the symmetric sum of multiple integrals driven by semimartingales. Under some additional assumptions on $H$ and $c$ we prove in Section 4 well-posedness of certain boundary value problems for stochastic Hamilton systems (1.2) for times $t$ not exceeding some stopping time. For proving this we first observe that well-posedness is equivalent to the statement that the map $p_{0} \mapsto X\left(t, t_{0}, x_{0}, p_{0}\right)$ (where $X\left(t, t_{0}, x_{0}, p_{0}\right)$ is a solution of (1.2) with initial conditions $\left(x_{0}, p_{0}\right)$ at time $\left.t=t_{0}\right)$ is a diffeomorphism. We call this statement diffeomorphism theorem. In Section 5 we develop the method of stochastic characteristics to solve stochastic HamiltonJacobi equations with Lévy noise.

Some applications of our results to the theory of stochastic heat equation (large deviation type asymptotics) are considered in the paper [KT2].

\section{Preliminaries}

Throughout this paper we consider Lévy processes as driving noise terms in the Hamiltonian system (1.2). Our standard references for Lévy processes are the monographs by Bertoin [Ber1] and Sato [Sa]. For Lévy processes and stochastic calculus with jumps we use the books by Jacod and Shiryaev [JSh] and Protter [Pro]. We will collect a few definitions and results from these books.

A Lévy process (on $\mathbb{R}^{d}$ ) is a stochastic process $\left\{\xi_{t}\right\}_{t \geqslant 0}$ on a probability space $(\Omega, \mathcal{F}, \mathbb{P})$ with stationary and independent increments which is also stochastically continuous. We will assume that $\xi_{0}=0$ a.s. The state space will always be $\mathbb{R}^{d}$. We can (and will) choose a version that has rightcontinuous sample paths with everywhere finite left-hand limits (càdlàg, for short); if not otherwise mentioned, we will use the augmented canonical filtration of $\left\{\xi_{t}\right\}_{t \geqslant 0}$. The process $\left\{\xi_{t}\right\}_{t \geqslant 0}$ is uniquely (up to stochastic equivalence) determined through its Fourier transform,

$$
\mathbb{E} e^{i \eta \xi_{t}}=e^{-t \psi(\eta)}, \quad t>0, \eta \in \mathbb{R}^{d}
$$

where the characteristic exponent $\psi: \mathbb{R}^{d} \rightarrow \mathbb{C}$ is given by the LévyKhinchine representation

$$
\psi(\eta)=i \ell \cdot \eta+\eta \cdot Q \eta+\int_{y \neq 0}\left(1-e^{i y \cdot \eta}+\frac{i y \cdot \eta}{1+|y|^{2}}\right) \nu(d y) .
$$


Here, $\ell$ is some vector in $\mathbb{R}^{d}, Q \in \mathbb{R}^{d \times d}$ is a positive semi-definite matrix and $\nu$ is the Lévy or jump measure with support in $\mathbb{R}^{d} \backslash\{0\}$ such that $\int_{y \neq 0}|y|^{2} \wedge 1 \nu(d y)<\infty$. The Lévy-Khinchine formula is actually a one-toone correspondence between the function $\psi$ and the Lévy triplet $(\ell, Q, \nu)$.

Stochastically, the Lévy-Khinchine representation translates into a path decomposition of the process $\left\{\xi_{t}\right\}_{t \geqslant 0}$. Fix some Borel set $A \subset \mathbb{R}^{d} \backslash\{0\}$, and write $N_{t}(\omega, A)$ for the Poisson point process with intensity measure $\nu(A)$. It is known that $N_{t}(\omega, A)$ describes jumps of $\xi_{t}$ with sizes contained in $A$ and we get

$$
\xi_{t}(\omega)=\alpha t+B_{t}(\omega)+M_{t}(\omega)+J_{t}(\omega),
$$

where $\alpha=\mathbb{E}\left(\xi_{1}-\int_{|y| \geqslant 1} y N_{1}(\omega, d y)\right)$ is the drift coefficient, $B_{t}$ is a $d$-dimensional Wiener process with (possibly degenerate) covariance matrix $Q$,

$$
M_{t}(\omega)=\int_{|y|<1} y\left(N_{t}(\omega, d y)-t \nu(d y)\right)
$$

is a martingale which is the compensated sum of all small jumps (modulus less than 1), and

$$
J_{t}(\omega)=\sum_{0<s \leqslant t} \Delta \xi_{s} \mathbf{1}_{\left\{\left|\Delta \xi_{s}\right| \geqslant 1\right\}}
$$

is the sum of all big jumps (modulus greater than 1). As usual, we write $\Delta \xi_{s}=\xi_{s}-\xi_{s-}=\xi_{s}-\lim _{r \uparrow s} \xi_{r}$ for the jump at time $s>0$. Note that $J_{t}$ is a process of bounded variation on compact time-intervals. This is the case since càdlàg paths can have only finitely many jumps of size $\geqslant 1$ on any finite time interval. The above decomposition of $\xi_{t}$ shows that Lévy processes are semimartingales and, therefore, good stochastic integrators.

The following two formulae for point processes hold whenever the righthand side is finite:

$$
\mathbb{E}\left(\int_{A} f(y) N_{t}(\cdot, d y)\right)=t \int_{A} f(y) \nu(d y)
$$

and

$$
\mathbb{E}\left(\left\{\int_{A} f(y)\left(N_{t}(\cdot, d y)-t \nu(d y)\right)\right\}^{2}\right)=t \int_{A} f(y)^{2} \nu(d y) .
$$

In particular, we get

$$
\mathbb{E}\left(\sum_{s \leqslant t} f\left(\Delta \xi_{s}\right)\right)=t \int f(y) \nu(d y)
$$

for finite right-hand sides. 
It is not hard to see that

$t \mapsto \xi_{t} \quad$ has a.s. finite variation if and only if $\int_{0<|y|<1}|y| \nu(d y)<\infty$ and that

$$
\mathbb{E}\left|\xi_{t}\right|<\infty \text { if and only if } \int_{|y| \geqslant 1}|y| \nu(d y)<\infty
$$

If $\xi_{t}$ has a.s. bounded jumps, i.e., if the support of $\nu$ is a bounded set, $\xi_{t}$ has absolute moments of any order.

We will also need the following simple Lemma. Since we could not find a precise reference for it, we include a short proof.

Lemma 2.1 Let $\left\{\xi_{t}\right\}_{t \geqslant 0}, \xi=\left(\xi_{1}, \ldots, \xi_{d}\right)$ be a Lévy process with $Q=0$ and Lévy measure $\nu$ satisfying $\int_{|y|>1}|y|^{2} \nu(d y)<\infty$. For any $0<\varepsilon<1 / 2$ we find a stopping time $\mathcal{R}_{\varepsilon}(\omega)<1$ such that

$$
\vartheta_{t}=2 \sum_{i=1}^{d}\left(\sup _{\tau \in[0, t]}\left|\xi_{i, \tau}\right|+\left[\xi_{i}, \xi_{i}\right]_{t}^{\frac{1}{2}}\right)<t^{\frac{1}{2}-\varepsilon}
$$

holds for all $t<\mathcal{R}_{\varepsilon}$, where $\mathbb{P}\left(\mathcal{R}_{\varepsilon}>0\right)=1$. In particular, one can find a stopping time $\mathcal{R}>0$ a.s. such that for all $t<\mathcal{R}$

$$
\vartheta_{t}=2 \sum_{i=1}^{d}\left(\sup _{\tau \in[0, t]}\left|\xi_{i, \tau}\right|+\left[\xi_{i}, \xi_{i}\right]_{t}^{\frac{1}{2}}\right)<1 .
$$

\section{Remarks}

1. In this paper we will use only the fact that $\vartheta_{t}<1$ for $t<\mathcal{R}$. The stopping times $\mathcal{R}_{\varepsilon}$ will be needed in [KT2].

2. Lemma 2.1 remains valid if $Q \neq 0$. Since we do not need this result, we settle for the case $Q=0$ and the somewhat simpler proof.

Proof. As usual we write $\xi_{t}^{*}=\sup _{\tau \in[0, t]}\left|\xi_{\tau}\right|$. Since $Q=0$, we get from (2.1)

$$
\mathbb{E}\left(\left\{\xi_{t}^{*}\right\}^{2}\right) \leqslant 3\left[|\alpha|^{2} t^{2}+\mathbb{E}\left(\left\{M_{t}^{*}\right\}^{2}\right)+\mathbb{E}\left(\left\{J_{t}^{*}\right\}^{2}\right)\right] .
$$

Using $(a+b)^{2} \leqslant 2 a^{2}+2 b^{2}$ and (2.2) we get for $t<1$

$$
\begin{aligned}
\mathbb{E}\left(\left\{J_{t}^{*}\right\}^{2}\right) & \leqslant \mathbb{E}\left(\left\{\sum_{s \leqslant t}\left|\Delta \xi_{s}\right| \mathbf{1}_{\left\{\left|\Delta \xi_{s}\right|>1\right\}}\right\}^{2}\right)=\mathbb{E}\left(\left\{\int_{|x|>1}|x| N_{t}(\cdot, d x)\right\}^{2}\right) \\
& \leqslant 2 \mathbb{E}\left(\left\{\int_{|x|>1}|x|\left(N_{t}(\cdot, d x)-t \nu(d x)\right)\right\}^{2}\right)+2 t^{2}\left(\int_{|x|>1}|x| \nu(d x)\right)^{2} \\
& \leqslant 2 t\left(1+\nu\left(B_{1}^{c}(0)\right)\right) \int_{|x|>1}|x|^{2} \nu(d x),
\end{aligned}
$$

where we used $t<1$ and Jensen's inequality for the last term. 
Doob's martingale inequality and (2.2) give

$$
\mathbb{E}\left(\left\{M_{t}^{*}\right\}^{2}\right) \leqslant 4 \mathbb{E}\left(M_{t}^{2}\right)=4 t \int_{|x|<1}|x|^{2} \nu(d x) .
$$

Formula (2.3) implies that

$$
\mathbb{E}\left([\xi, \xi]_{t}\right)=t \int_{|x|>0}|x|^{2} \nu(d x) .
$$

Thus, the process

$$
\zeta_{t}=\left\{\xi_{t}^{*}\right\}^{2}+[\xi, \xi]_{t}
$$

satisfies $\mathbb{E} \zeta_{t}<C t$ for $t<1$, where $C=C(\alpha, \nu)>0$ is a constant. By Chebyshev's inequality

$$
\mathbb{P}\left(\zeta_{t}>R\right) \leqslant \frac{\mathbb{E}\left(\zeta_{t}\right)}{R} \leqslant \frac{C t}{R}, \quad t<1 .
$$

Choosing $t=2^{-k}$ and $R=(8 d)^{-1} 2^{-(1-\varepsilon) k}$ we find

$$
\sum_{k=1}^{\infty} \mathbb{P}\left\{\zeta_{2^{-k}}>(8 d)^{-1} 2^{-(1-\varepsilon) k}\right\} \leqslant 8 d C \sum_{k=1}^{\infty} 2^{-\varepsilon k}<\infty .
$$

The Borel-Cantelli Lemma implies that

$$
\zeta_{2^{-k}} \leqslant(8 d)^{-1} 2^{-(1-\varepsilon) k} \quad \text { for } \quad k>k_{0}(\omega) \text { for some } k_{0}(\omega) \in \mathbb{N} .
$$

Set $k_{1}(\omega)=k_{0}(\omega) \vee\left[\frac{(1-2 \varepsilon)}{\varepsilon}+1\right]$. Then $(1-2 \varepsilon) \frac{k+1}{k} \leqslant 1-\varepsilon$ for $k>k_{1}(\omega)$. If $2^{-(k+1)} \leqslant t<2^{-k}$ for some $k>k_{1}(\omega)$ we find, as $t \mapsto \zeta_{t}$ is an increasing function,

$(8 d) \zeta_{t} \leqslant(8 d) \zeta_{2^{-k}} \leqslant 2^{-(1-\varepsilon) k} \leqslant\left(2^{-(k+1)}\right)^{\frac{(1-\varepsilon) k}{k+1}} \leqslant\left(2^{-(k+1)}\right)^{(1-2 \varepsilon)} \leqslant t^{(1-2 \varepsilon)}$.

Using the elementary inequality $\left(a_{1}+\cdots+a_{2 d}\right)^{2} \leqslant(2 d)\left(a_{1}^{2}+\cdots+a_{2 d}^{2}\right)$ we get with $\mathcal{R}_{\varepsilon}(\omega)=2^{-k_{1}(\omega)}$

$$
\vartheta_{t}^{2} \leqslant(8 d) \zeta_{t} \leqslant t^{1-2 \varepsilon}, \quad \forall t<\mathcal{R}_{\varepsilon},
$$

and the lemma follows.

Notation. Most of our notation should be standard or self-explanatory. All stochastic integrals are Itô-integrals and our main reference texts for stochastic integrals with jumps are Jacod and Shiryaev [JSh] and Protter [Pro]. In particular, we follow the conventions of Protter

$$
\int_{a}^{b} X_{s} d Y_{s}:=\int_{(a, b]} X_{s} d Y_{s}, \quad[X, Y]_{0}=X_{0} Y_{0} \quad \text { and } \quad X_{0-}=\left(X_{-}\right)_{0}=0
$$




\section{Estimates for multiple stochastic integrals driven by semimartingales}

Here we derive some estimates for multiple stochastic integrals which will be needed later on. We use the following notation. For any $A \in \mathbb{R}^{M \times N}$ we write

$$
\|A\|_{\infty}=\max _{\substack{i=1, \ldots, M \\ j=1, \ldots, N}}\left|(A)_{i, j}\right| .
$$

We will always consider $k$-fold stochastic integrals driven by (general) real-valued semimartingales $\left\{\eta_{j, t}\right\}_{t \geqslant 0}, \quad j=1, \ldots, d$, with càdlàg paths or by the deterministic process $\eta_{0, t}=t$. We assume that all semimartingales are on the same probability space $(\Omega, \mathcal{F}, \mathbb{P})$ and are adapted to the same filtration $\left\{\mathcal{F}_{t}\right\}_{t \geqslant 0}$. The filtration is assumed to satisfy the usual conditions, i.e., it is right-continuous and augmented. Since the $d \eta_{j, t}, j=0, \ldots, d$, may appear in any order we want to keep track when we deal with a Stieltjes differential $d \tau=d \eta_{0, \tau}$ and a (genuinely) stochastic differential $d \eta_{j, \tau}, j=1, \ldots, d$. To do so we introduce and fix throughout this section a sequence $\ell_{n} \in \mathbb{N}$ such that

$$
\ell_{2 n-1} \leqslant \ell_{2 n} \quad \text { and } \quad \ell_{2 n}+1<\ell_{2 n+1} .
$$

We set

$$
\mathcal{M}_{i}= \begin{cases}\{1, \ldots, d\} & \text { if } \ell_{2 n-1} \leqslant i \leqslant \ell_{2 n} \\ \{0\} & \text { otherwise. }\end{cases}
$$

This means that for $\left(j_{1}, \ldots, j_{k}\right) \in \mathcal{M}=\mathcal{M}_{1} \times \cdots \times \mathcal{M}_{k}$ the first $\ell_{1}-1$ integrals of the stochastic differential

$$
d \eta_{j_{1}, \tau_{1}} d \eta_{j_{2}, \tau_{2}} \ldots d \eta_{j_{k}, \tau_{k}}
$$

are deterministic, the next up to label $\ell_{2}$ are semimartingales, those up to $\ell_{3}-1$ are again deterministic, etc.:

$$
\begin{aligned}
\ell_{1} & =\min \left\{s \in[0, k]: j_{s} \neq 0\right\} \\
\ell_{2} & =\min \left\{s \in\left(\ell_{1}, k\right]: j_{s}=0\right\}-1 \\
& \vdots \\
\ell_{2 n-1} & =\min \left\{s \in\left(\ell_{2 n-2}, k\right]: j_{s} \neq 0\right\} \\
\ell_{2 n} & =\min \left\{s \in\left(\ell_{2 n-1}, k\right]: j_{s}=0\right\}-1
\end{aligned}
$$

$(\min \emptyset=k+1)$. Moreover,

$$
\mathfrak{m}=\mathfrak{m}(k)=\sum_{n \in \mathbb{N}, \ell_{2 n} \leqslant k}\left(\ell_{2 n}-\ell_{2 n-1}+1\right)
$$

is the number of non-trivial differentials in (3.2), i.e.

$$
\mathfrak{m}=\#\left\{s: j_{s} \neq 0\right\} .
$$


Let $W_{j, \tau}=W_{j, \tau}(\omega), I_{0, \tau}=I_{0, \tau}(\omega)$ be $\mathbb{R}^{M \times M_{\text {-valued continuous processes }}}$ adapted to the filtration $\left\{\mathcal{F}_{\tau}\right\}_{\tau \geqslant 0}$ and such that

$$
\sup _{s \geqslant t_{0}}\left\|W_{j, s}\right\|_{\infty} \leqslant 1, \quad j=0, \ldots, d
$$

and let $v_{\tau}$ be some real-valued $\mathcal{F}_{t}$-adapted increasing process such that for any $s, t \in \mathbb{R}_{+}, s<t$,

$$
\left\|W_{j, t}-W_{j, s}\right\|_{\infty} \leqslant v_{t}-v_{s}, \quad j=1, \ldots, d .
$$

We also assume that $\left[\eta_{i}, \eta_{i}\right]^{c}=0, \quad i=1, \ldots, d$, and

$$
2 \sup _{t_{0} \leqslant \tau_{1} \leqslant \tau}\left|\eta_{i, \tau_{1}}-\eta_{i, t_{0}}\right| \leqslant 1, \quad i=1, \ldots, d .
$$

Notice that the assumptions (3.4), (3.6) can always be achieved by suitable (pre-)stopping arguments.

For $0 \leqslant t_{0} \leqslant \tau$ we set

$$
=\sum_{\left(j_{1}, \ldots, j_{k}\right) \in \mathcal{M}} \int_{t_{0}}^{\tau} W_{j_{k}, \tau_{k}}\left(\int_{t_{0}}^{\tau_{k}-} \cdots\left(\int_{t_{0}}^{\tau_{2}-} W_{j_{1}, \tau_{1}} I_{0, \tau_{1}} d \eta_{j_{1}, \tau_{1}}\right) \ldots d \eta_{j_{k-1}, \tau_{k-1}}\right) d \eta_{j_{k}, \tau_{k}}
$$

where $\mathcal{M}=\mathcal{M}_{1} \times \cdots \times \mathcal{M}_{k}$ as above, and

$$
D_{\tau}=M \cdot d\left[v_{\tau}-v_{t_{0}}+4 \sum_{j=1}^{d}\left(\sup _{t_{0} \leqslant \tau_{1} \leqslant \tau}\left|\eta_{j, \tau_{1}}-\eta_{j, t_{0}}\right|+\left(\left[\eta_{j}, \eta_{j}\right]_{\tau}-\left[\eta_{j}, \eta_{j}\right]_{t_{0}}\right)^{\frac{1}{2}}\right)\right] .
$$

We will use the abbreviation $B V$-process for a process with (almost surely) paths of bounded variation on compact time-intervals.

We can now state the main result of this section.

Proposition 3.1 Let $\eta_{i, \tau}, i=1, \ldots, d$, be $\mathcal{F}_{\tau}$-semimartingales, $W_{j, \tau}$ be $\mathcal{F}_{\tau}$-adapted continuous processes as set out above, $\ell_{n} \in \mathbb{N}$ be any fixed sequence such that (3.1) holds and $I_{k, \tau}$ be as defined above in (3.7). Moreover we assume that

$$
W_{j, \tau} W_{i, \tau}=W_{i, \tau} W_{j, \tau} \quad j, i=1, \ldots, d .
$$

If $I_{0, \tau}=E_{M} \in \mathbb{R}^{M \times M}$ is the identity matrix or if $\ell_{1}>1$ (or both), we have

$$
\left\|I_{k, \tau}\right\|_{\infty} \leqslant b_{k} D_{\tau}^{\mathfrak{m}}\left\{M\left(\tau-t_{0}\right)\right\}^{k-\mathfrak{m}}\left\|I_{0, \tau}^{*}\right\|_{\infty},
$$

where $\left\|I_{0, \tau}^{*}\right\|_{\infty}=\sup _{t_{0} \leqslant s \leqslant \tau}\left\|I_{0, s}\right\|_{\infty}$, with $\mathfrak{m}$ from (3.3) and

$$
b_{k}=\frac{\left(2^{7}\right)^{k}}{(\ln \{\ln \{k+2\}\})^{\frac{k}{16}}} .
$$


The proof of Proposition 3.1 is quite technical and a few words of guidance are in order. Basically, we prove Proposition 3.1 by induction in $k$, the number of iterated integrals in $I_{k}$. There are two different ways how $I_{k+1}$ can arise from $I_{k}:(\mathbf{i})$ the $(k+1)$ st integrator is deterministic, or (ii) the $(k+1)$ st integrator is a semimartingale. Case (i) is easily dealt with (Case 1 of Step 1 of the proof), while (ii) requires a few auxiliary results which we prove in Lemmas 3.1-3.6 below. The idea here is to consider the last two, i.e. $k$ th and $(k+1)$ st, integrations simultaneously and to look first at the more complicated (but symmetrised) object

$$
z_{k+1, \tau}^{\widehat{m}}=\sum_{i=1}^{d} \sum_{J \in \mathbb{N}_{0}^{d},|J|=\widehat{m}} \sum_{J^{\prime} \leqslant J} \Re_{J^{\prime}, J, \tau} \int_{0}^{\tau} U_{J, \tau_{2}}\left(\int_{0}^{\tau_{2}-} W_{i, \tau_{1}} I_{k-1, \tau_{1}-} d \eta_{i, \tau_{1}}\right) d \eta_{\tau_{2}}^{J^{\prime}},
$$

where $U_{J, \tau}$ is a product of $W_{j}$ 's and $\mathfrak{R}_{J^{\prime}, J, \tau}$ are the coefficients of a multinomial series (see (3.19) and (3.20)). Notice that $I_{k+1, \tau}=z_{k+1, \tau}^{1}$. The symmetrisation has the effect that we are effectively integrating against $d\left(\eta_{\bullet}-\eta_{\tau}\right)^{J}$ - but this integrator would not be well-defined in Itô's sense.

The technique is to apply integration by parts to the two inner integrals in (3.12) (the general formula is given in Lemma 3.1, Corollary 3.1 contains the 'symmetrised' version which we are going to use later on) and then to estimate the three appearing terms (3.27)-(3.29) which is done in Lemma 3.3. Depending on the nature of the integrators in (3.12), the main estimates are done in Lemmas 3.5 and 3.6. Their proofs use mainly Lemmas 3.2-3.4 which are of technical nature and can be skipped on first reading. This induction gives the basic estimate of Proposition 3.1 without revealing the form of the coefficients $b_{k}$. The coefficients are obtained in a separate induction (Step 2 in the proof of Proposition 3.1). This part of the proof rests entirely on some tedious estimates and recurrence relations which we deferred to the appendix (Lemmas A.1-A.4).

\section{Notation}

(i) We will use throughout the paper matrix and vector notation for stochastic integrals. Since matrices are, in general, non-commutative the position of stochastic differentials etc. is important.

(ii) All stochastic integrals, where the integrand is a vector (or a matrix) and the integrator is an $\mathbb{R}$-valued semimartingale will be understood coordinate-wise. In a similar way, brackets of vectors and $\mathbb{R}$-semimartingales or matrices of $\mathbb{R}$-semimartingales are understood coordinatewise. The bracket of two matrices $A, B$ is defined as a matrix

$$
[A, B]_{i k}=\sum_{j=1}^{M}\left[a_{i j}, b_{j k}\right], \quad i, k=1, \ldots, M,
$$

which is compatible with the rules of stochastic calculus and matrix algebra. 
We start with rewriting the integration by parts formula in the form we need later on.

Lemma 3.1 Let $\left\{U_{\tau}\right\}_{\tau \geqslant 0}$ be an $\mathcal{F}_{\tau}$-adapted continuous $B V$ process with values in $\mathbb{R}^{M \times M},\left\{\Phi_{\tau}\right\}_{\tau \geqslant 0}$ an $\mathcal{F}_{\tau}$-adapted càdlàg process with values in $\mathbb{R}^{M \times M}$ and let $\left\{\nu_{\tau}\right\}_{\tau \geqslant 0},\left\{\kappa_{\tau}\right\}_{\tau \geqslant 0}$ be two real-valued $\mathcal{F}_{\tau}$-semimartingales. Then the iterated stochastic integral

$$
Q_{\tau}=\int_{0}^{\tau} U_{\tau_{2}}\left(\int_{0}^{\tau_{2}-} \Phi_{\tau_{1}-} d \nu_{\tau_{1}}\right) d \kappa_{\tau_{2}}
$$

can be written in the form

$$
\begin{aligned}
Q_{\tau}=\kappa_{\tau} \int_{0}^{\tau} d U_{\tau_{2}} & \times \int_{0}^{\tau_{2}-} \Phi_{\tau_{1}-} d \nu_{\tau_{1}}+\kappa_{\tau} \int_{0}^{\tau} U_{\tau_{2}} \Phi_{\tau_{2}-} d \nu_{\tau_{2}}-\int_{0}^{\tau} U_{\tau_{2}} \Phi_{\tau_{2}-} d[\nu, \kappa]_{\tau_{2}} \\
& -\int_{0}^{\tau} d U_{\tau_{2}} \times \kappa_{\tau_{2}-} \int_{0}^{\tau_{2}-} \Phi_{\tau_{1}-} d \nu_{\tau_{1}}-\int_{0}^{\tau} \kappa_{\tau_{2}-} U_{\tau_{2}} \Phi_{\tau_{2}-} d \nu_{\tau_{2}} .
\end{aligned}
$$

Proof. We use the following integration by parts formula for $\mathbb{R}$-valued semimartingales:

$$
\int_{0}^{\tau} Y_{\tau_{2}-} d Z_{\tau_{2}}=Y_{\tau} Z_{\tau}-Y_{0} Z_{0}-[Y, Z]_{\tau}-\int_{0}^{\tau} Z_{\tau_{2}-} d Y_{\tau_{2}}
$$

With the coordinate conventions detailed in the above remark we may choose

$$
Y_{\tau_{2}}=U_{\tau_{2}} \int_{0}^{\tau_{2}} \Phi_{\tau_{1}-} d \nu_{\tau_{1}} \quad \text { and } \quad Z_{\tau_{2}}=\kappa_{\tau_{2}}
$$

Clearly, $Y_{0}=0$ and therefore

$$
\begin{aligned}
Q_{\tau} & =\kappa_{\tau} U_{\tau} \int_{0}^{\tau} \Phi_{\tau_{1}-} d \nu_{\tau_{1}}-\left[U \bullet \int_{0}^{\bullet} \Phi_{\tau_{1}-} d \nu_{\tau_{1}}, \kappa_{\bullet}\right]_{\tau}-\int_{0}^{\tau} \kappa_{\tau_{2}-} d\left(U_{\tau_{2}} \int_{0}^{\tau_{2}} \Phi_{\tau_{1}-} d \nu_{\tau_{1}}\right) \\
& =\mathbf{I}+\mathbf{I I}+\mathbf{I I I} .
\end{aligned}
$$

An application of Itô's formula gives

$$
\begin{aligned}
& d\left(U_{\tau_{2}} \int_{0}^{\tau_{2}} \Phi_{\tau_{1}-} d \nu_{\tau_{1}}\right)= \\
& =d U_{\tau_{2}} \times \int_{0}^{\tau_{2}-} \Phi_{\tau_{1}-} d \nu_{\tau_{1}}+U_{\tau_{2}} \Phi_{\tau_{2}-} d \nu_{\tau_{2}}+d\left[U_{\bullet}, \int_{0}^{\bullet} \Phi_{\tau_{1}-} d \nu_{\tau_{1}}\right]_{\tau_{2}}
\end{aligned}
$$

Since $U$ is a continuous BV-process, the square bracket vanishes and we get

$$
\mathbf{I}=\kappa_{\tau} \int_{0}^{\tau} d U_{\tau_{2}} \times \int_{0}^{\tau_{2}-} \Phi_{\tau_{1}-} d \nu_{\tau_{1}}+\kappa_{\tau} \int_{0}^{\tau} U_{\tau_{2}} \Phi_{\tau_{2}-} d \nu_{\tau_{2}} .
$$


From (3.14) we also find

$$
\begin{aligned}
\mathbf{I I} & =-\left[\left(U \bullet \int_{0}^{\bullet} \Phi_{\tau_{1}-} d \nu_{\tau_{1}}\right), \kappa_{\bullet}\right]_{\tau} \\
& =-\left[\left(\int_{0}^{\bullet} U_{\tau_{2}} \Phi_{\tau_{2}-} d \nu_{\tau_{2}}\right), \kappa_{\bullet}\right]_{\tau}-\left[\int_{0}^{\bullet} d U_{\tau_{1}} \times \int_{0}^{\tau_{1}-} \Phi_{\tau_{2}-} d \nu_{\tau_{2}}, \kappa_{\bullet}\right]_{\tau}
\end{aligned}
$$

Since $U_{\bullet}$ is a continuous BV-process, so is the stochastic integral driven by $d U$, and the last bracket above vanishes. So,

$$
\mathbf{I I}=-\int_{0}^{\tau} U_{\tau_{2}} \Phi_{\tau_{2}-} d[\nu, \kappa]_{\tau_{2}}
$$

Finally, using (3.14) the third time gives

$$
\mathbf{I I I}=-\int_{0}^{\tau} \kappa_{\tau_{2}-} d U_{\tau_{2}} \times \int_{0}^{\tau_{2}-} \Phi_{\tau_{1}-} d \nu_{\tau_{1}}-\int_{0}^{\tau} \kappa_{\tau_{2}-} U_{\tau_{2}} \Phi_{\tau_{2}-} d \nu_{\tau_{2}}
$$

Combining this with (3.15) and (3.16) completes the proof.

Later on, we will use Lemma 3.1 in the following form.

Corollary 3.1 Let $\left\{U_{\tau}\right\}_{\tau \geqslant 0},\left\{\Phi_{\tau}\right\}_{\tau \geqslant 0},\left\{\nu_{\tau}\right\}_{\tau \geqslant 0}$ be as in Lemma 3.1 and let $\left\{\widetilde{\kappa}_{\alpha, \tau}\right\}_{\tau \geqslant 0}$ and $\left\{\kappa_{\alpha, \tau}\right\}_{\tau \geqslant 0}$ be finitely many $(\alpha \in \mathfrak{A})$ real-valued $\mathcal{F}_{\tau}$-semimartingales such that

$$
\sum_{\alpha \in \mathfrak{A}} \widetilde{\kappa}_{\alpha, \tau} \kappa_{\alpha, \tau}=0
$$

Then the iterated stochastic integral

$$
Q_{\tau}=\sum_{\alpha \in \mathfrak{A}} \widetilde{\kappa}_{\alpha, \tau} \int_{0}^{\tau} U_{\tau_{2}}\left(\int_{0}^{\tau_{2}-} \Phi_{\tau_{1}-} d \nu_{\tau_{1}}\right) d \kappa_{\alpha, \tau_{2}}
$$

can be written in the form

$$
\begin{aligned}
& Q_{\tau}=-\sum_{\alpha \in \mathfrak{A}} \widetilde{\kappa}_{\alpha, \tau} \int_{0}^{\tau} U_{\tau_{2}} \Phi_{\tau_{2}-} d\left[\nu, \kappa_{\alpha}\right]_{\tau_{2}}-\sum_{\alpha \in \mathfrak{A}} \widetilde{\kappa}_{\alpha, \tau} \int_{0}^{\tau} d U_{\tau_{2}} \times \kappa_{\alpha, \tau_{2}-} \int_{0}^{\tau_{2}-} \Phi_{\tau_{1}-} d \nu_{\tau_{1}} \\
& \quad-\sum_{\alpha \in \mathfrak{A}} \widetilde{\kappa}_{\alpha, \tau} \int_{0}^{\tau} \kappa_{\alpha, \tau_{2}-} U_{\tau_{2}} \Phi_{\tau_{2}-} d \nu_{\tau_{2}} .
\end{aligned}
$$

Proof. For a fixed $\alpha \in \mathfrak{A}$ we can apply Lemma 3.1 with $\kappa_{\tau}=\kappa_{\alpha, \tau}$ to get (3.13). Now multiply (3.13) with $\widetilde{\kappa}_{\alpha, \tau}$ and sum over $\alpha \in \mathfrak{A}$. Because of (3.17), the first two terms vanish and we get (3.18). 
Recall the following common notations for multi-indices $J=\left(j_{1}, \ldots, j_{d}\right)$, $J^{\prime}=\left(j_{1}^{\prime}, \ldots, j_{d}^{\prime}\right) \in \mathbb{N}_{0}^{d}$ : we say $J^{\prime} \leqslant J$ if $j_{1}^{\prime} \leqslant j_{1}, \ldots, j_{d}^{\prime} \leqslant j_{d},|J|=j_{1}+\cdots+j_{d}$, $J \pm J^{\prime}=\left(j_{1} \pm j_{1}^{\prime}, \ldots, j_{d} \pm j_{d}^{\prime}\right)$, and if $\eta_{\tau}=\left(\eta_{1, \tau}, \ldots, \eta_{d, \tau}\right)$,

$$
\eta_{\tau}^{J}=\eta_{1, \tau}^{j_{1}} \ldots \eta_{d, \tau}^{j_{d}}
$$

We also set

$$
\Re_{J^{\prime}, J, \tau}=(-1)^{|J|-\left|J^{\prime}\right|}\left(\begin{array}{c}
j_{1} \\
j_{1}^{\prime}
\end{array}\right) \ldots\left(\begin{array}{c}
j_{d} \\
j_{d}^{\prime}
\end{array}\right) \eta_{\tau}^{J-J^{\prime}} \quad J^{\prime} \leqslant J .
$$

From the binomial formula we easily see

$$
\sum_{J^{\prime} \leqslant J} \mathfrak{R}_{J^{\prime}, J, \tau} \eta_{\tau_{2}}^{J^{\prime}}=\left(\eta_{\tau_{2}}-\eta_{\tau}\right)^{J}=\left(\eta_{1, \tau_{2}}-\eta_{1, \tau}\right)^{j_{1}} \ldots\left(\eta_{d, \tau_{2}}-\eta_{d, \tau}\right)^{j_{d}} .
$$

Below is a technical lemma which we need in order to estimate the norm of some integrals driven by BV-processes.

Lemma 3.2 Let $\left\{\eta_{i, \tau}\right\}_{\tau \geqslant 0}, \quad i=1, \ldots, d$, be real-valued $\mathcal{F}_{\tau}$-semimartingales with $\left[\eta_{i}, \eta_{i}\right]^{c}=0, \eta_{i, 0}=0$ and such that the inequalities (3.6) are satisfied. Then we have for all $J^{\prime}, J \in \mathbb{N}_{0}^{d}$ with $J^{\prime} \leqslant J$ and $\left|J^{\prime}\right|=m^{\prime},|J|=m$

$$
\begin{aligned}
& \text { (i) } \sup _{0 \leqslant s \leqslant \tau}\left|\eta_{s}^{J-J^{\prime}}\right| \leqslant(4 M \cdot d)^{-\left(m-m^{\prime}\right)+1} D_{\tau}^{m-m^{\prime}-1}, \\
& (\boldsymbol{i i}) \sup _{0 \leqslant s \leqslant \tau}\left|\eta_{s}^{J-J^{\prime}}\right| \leqslant(4 M \cdot d)^{-\left(m-m^{\prime}\right)} D_{\tau}^{m-m^{\prime}}, \\
& (\boldsymbol{i i i}) \sup _{0 \leqslant s \leqslant \tau}\left|\eta_{s}^{J^{\prime}}\right| \leqslant(4 M \cdot d)^{-m^{\prime}} D_{\tau}^{m^{\prime}}, \\
& (\boldsymbol{i v})\left|\Delta\left[\eta^{J^{\prime}}, \eta_{i}\right]_{\tau}\right| \leqslant(2 M \cdot d)^{-m^{\prime}-1} D_{\tau}^{m^{\prime}} \Delta D_{\tau}, \\
& \text { (v) } \sum_{J^{\prime} \leqslant J}\left|\Re_{J^{\prime}, J, \tau}\right| \sup _{0 \leqslant \tau_{2} \leqslant \tau}\left|\Delta\left[\eta^{J^{\prime}}, \eta_{i}\right]_{\tau_{2}}\right| \leqslant 2^{-1}(M \cdot d)^{-m-1} D_{\tau}^{m} \Delta D_{\tau}, \\
& \text { (vi) } \sum_{J^{\prime} \leqslant J}\left|\Re_{J^{\prime}, J, \tau}\right| \sup _{0 \leqslant \tau_{2} \leqslant \tau}\left|\eta_{\tau_{2}}^{J^{\prime}}\right| \leqslant 2(2 M \cdot d)^{-m+1} D_{\tau}^{m-1}, \\
& \text { (vii) } \sum_{J^{\prime} \leqslant J}\left|\Re_{J^{\prime}, J, \tau}\right|(2 M \cdot d)^{-m^{\prime}-1} D_{\tau}^{m^{\prime}} \leqslant(M \cdot d)^{-m-1} D_{\tau}^{m} .
\end{aligned}
$$

Proof. By the very definition (3.8) of $D_{\tau}$ we get

$$
\max _{i=1, \ldots, d} \max \left\{\left|\eta_{i, \tau}\right|,\left|\Delta \eta_{i, \tau}\right|\right\} \leqslant(4 M \cdot d)^{-1} D_{\tau} .
$$

Hence, due to (3.6),

$$
\begin{aligned}
\sup _{0 \leqslant s \leqslant \tau}\left|\eta_{s}^{J-J^{\prime}}\right| \leqslant \sup _{0 \leqslant s \leqslant \tau} \max _{i=1, \ldots, d}\left|\eta_{i, s}\right|^{m-m^{\prime}} & \leqslant \sup _{0 \leqslant s \leqslant \tau} \max _{i=1, \ldots, d}\left|\eta_{i, s}\right|^{m-m^{\prime}-1} \\
& \leqslant(4 M \cdot d)^{-\left(m-m^{\prime}\right)+1} D_{\tau}^{m-m^{\prime}-1}
\end{aligned}
$$

and (3.2) follows. Similarly we obtain (3.2) and (3.2). 
We proceed with (3.2). Using (3.21), the elementary identity $\Delta\left(a_{1} \cdot a_{2} \cdot \ldots \cdot a_{m^{\prime}}\right)=\sum_{r=1}^{m^{\prime}} a_{0} \ldots a_{r-1}\left(\Delta a_{r}\right)\left(a_{r+1}-\Delta a_{r+1}\right) \ldots\left(a_{m^{\prime}+1}-\Delta a_{m^{\prime}+1}\right)$, where $a_{0}=a_{m^{\prime}+1}=1, \Delta a_{0}=\Delta a_{m^{\prime}+1}=0$, and $m^{\prime} 2^{-m^{\prime}+1} \leqslant 1$ we find

$$
\begin{aligned}
\left|\Delta \eta_{\tau}^{J^{\prime}}\right| & \leqslant m^{\prime}\left(\sup _{0 \leqslant s \leqslant \tau} \max _{i=1, \ldots, d}\left|\eta_{i, s}\right|\right)^{m^{\prime}-1}\left(\max _{i=1, \ldots, d}\left|\Delta \eta_{i, \tau}\right|\right) \\
& \leqslant(2 M \cdot d)^{-m^{\prime}+1} D_{\tau}^{m^{\prime}-1} \max _{i=1, \ldots, d}\left|\Delta \eta_{i, \tau}\right| .
\end{aligned}
$$

Since $\eta_{i}$ and $\eta^{J}$ are pure jump semimartingales, it follows

$$
\left|\Delta\left[\eta^{J^{\prime}}, \eta_{i}\right]_{\tau}\right|=\left|\Delta \eta_{\tau}^{J^{\prime}} \Delta \eta_{i, \tau}\right| \leqslant(2 M \cdot d)^{-m^{\prime}+1} D_{\tau}^{m^{\prime}-1} \max _{i=1, \ldots, d}\left|\Delta \eta_{i, \tau}\right|^{2} .
$$

Due to $(3.8)$

$$
\Delta\left[\eta_{i}, \eta_{i}\right]_{\tau}^{\frac{1}{2}} \leqslant(4 M \cdot d)^{-1} \Delta D_{\tau}
$$

for any $i=1, \ldots, d$, where we used that $v_{\tau}$ and $\sup _{0 \leqslant s \leqslant \tau}\left|\eta_{s}\right|$ are increasing functions of $\tau$. Since

$$
\Delta\left(\left[\eta_{i}, \eta_{i}\right]_{\tau}^{\frac{1}{2}}\right)=\frac{\Delta\left[\eta_{i}, \eta_{i}\right]_{\tau}}{\left[\eta_{i}, \eta_{i}\right]_{\tau}^{\frac{1}{2}}+\left[\eta_{i}, \eta_{i}\right]_{\tau-}^{\frac{1}{2}}} \geqslant \frac{\Delta\left[\eta_{i}, \eta_{i}\right]_{\tau}}{2\left[\eta_{i}, \eta_{i}\right]_{\tau}^{\frac{1}{2}}} \geqslant \frac{\left|\Delta \eta_{i, \tau}\right|^{2}}{(2 M \cdot d)^{-1} D_{\tau}},
$$

we conclude

$$
\left|\Delta \eta_{i, \tau}\right|^{2} \leqslant(2 M \cdot d)^{-2} D_{\tau} \Delta D_{\tau}
$$

and the combination of (3.22), (3.23) proves (3.2).

Using the fact that

$$
\sum_{J^{\prime} \leqslant J}\left(\begin{array}{l}
j_{1} \\
j_{1}^{\prime}
\end{array}\right) \cdots\left(\begin{array}{l}
j_{d} \\
j_{d}^{\prime}
\end{array}\right)=2^{m}
$$

we see the implications: (3.2), (3.2) $\Longrightarrow(3.2) ; \quad(3.2),(3.2) \Longrightarrow(3.2)$; and $(3.2) \Longrightarrow(3.2)$.

Given $L^{\prime}=\left(l_{1}^{\prime}, \ldots, l_{d}^{\prime}\right) \in \mathbb{N}_{0}^{d}$ and $J=\left(j_{1}, \ldots, j_{d}\right) \in \mathbb{N}_{0}^{d}$ we put

$$
\begin{gathered}
f_{L^{\prime}}\left(x_{1}, \ldots, x_{d}\right)=x_{1}^{l_{1}^{\prime}} \ldots x_{d}^{l_{d}^{\prime}}, \\
\delta_{L^{\prime}, \tau_{2}}=\Delta\left[f_{L^{\prime}}(\eta)\right]_{\tau_{2}}-\sum_{i=1}^{d} \frac{\partial f_{L^{\prime}}\left(\eta_{\tau_{\tau_{2}-}}\right)}{\partial x_{i}} \Delta \eta_{i, \tau_{2}}
\end{gathered}
$$

and

$$
U_{J, \tau_{2}}=\frac{1}{J !}\left(W_{1, \tau_{2}}\right)^{j_{1}} \ldots\left(W_{d, \tau_{2}}\right)^{j_{d}}, \quad J !=j_{1} ! \ldots j_{d} !
$$


We are going to estimate the norms of the following integrals and sums

$$
\begin{aligned}
A_{J, i, \tau} & =-\sum_{J^{\prime} \leqslant J} \Re_{J^{\prime}, J, \tau} \int_{0}^{\tau} U_{J, \tau_{1}} W_{i, \tau_{1}} I_{k-1, \tau_{1}-} d\left[\eta^{J^{\prime}}, \eta_{i}\right]_{\tau_{1}} \\
B_{J, i, \tau} & =-\sum_{J^{\prime} \leqslant J} \Re_{J^{\prime}, J, \tau} \int_{0}^{\tau} d U_{J, \tau_{2}} \times \eta_{\tau_{2}-}^{J^{\prime}} \int_{0}^{\tau_{2}-} W_{i, \tau_{1}} I_{k-1, \tau_{1}-} d \eta_{i, \tau_{1}} \\
C_{L, \tau} & =\sum_{L^{\prime} \leqslant L} \sum_{0<\tau_{2} \leqslant \tau} \Re_{L^{\prime}, L, \tau} U_{L, \tau_{2}} I_{k-1, \tau_{2}-} \delta_{L^{\prime}, \tau_{2}}
\end{aligned}
$$

driven by BV-processes. As we will see in Lemmas 3.5 and 3.6, these integrals arise from an application of the integration by parts formula to iterated integrals of a certain type.

Lemma 3.3 For $m, k \in \mathbb{N}$ and $\mathcal{B}_{m}=\left\{J \in \mathbb{N}_{0}^{d}:|J|=m\right\}$ we have

$$
\begin{aligned}
& \left\|\sum_{i=1}^{d} \sum_{J \in \mathcal{B}_{m}} A_{J, i, \tau}\right\|_{\infty} \leqslant \frac{1}{2 m !} D_{\tau}^{m} \int_{0}^{\tau}\left\|I_{k-1, \tau_{1}-}\right\|_{\infty} d D_{\tau_{1}}, \\
& \left\|\sum_{i=1}^{d} \sum_{J \in \mathcal{B}_{m}} B_{J, i, \tau}\right\|_{\infty} \leqslant \frac{1}{(m-1) !} D_{\tau}^{m-1} \int_{0}^{\tau}\left\|I_{k, \tau_{2}-}\right\|_{\infty} d D_{\tau_{2}}, \\
& \left\|\sum_{L \in \mathcal{B}_{m+1}} C_{L, \tau}\right\|_{\infty} \leqslant \frac{1}{2 m !} D_{\tau}^{m} \int_{0}^{\tau}\left\|I_{k-1, \tau_{1}-}\right\|_{\infty} d D_{\tau_{1}} .
\end{aligned}
$$

Proof. Applying (3.2) of Lemma 3.2 we have

$$
\begin{aligned}
\left\|A_{J, i, \tau}\right\|_{\infty} & =\left\|\sum_{J^{\prime} \leqslant J} \Re_{J^{\prime}, J, \tau} \int_{0}^{\tau} U_{J, \tau_{1}} W_{i, \tau_{1}} I_{k-1, \tau_{1}-} d\left[\eta^{J^{\prime}}, \eta_{i}\right]_{\tau_{1}}\right\|_{\infty} \\
& \leqslant 2^{-1}(M \cdot d)^{-m-1} D_{\tau}^{m} \int_{0}^{\tau}\left\|U_{J, \tau_{1}} W_{i, \tau_{1}} I_{k-1, \tau_{1}-}\right\|_{\infty} d D_{\tau_{1}} .
\end{aligned}
$$

Because of

$$
\|Y Z\|_{\infty} \leqslant M\|Y\|_{\infty}\|Z\|_{\infty}, \quad Y, Z \in \mathbb{R}^{M \times M}
$$

definition (3.26), and condition (3.4) we obtain for $J \in \mathcal{B}_{m}$

$$
\left\|U_{J, \tau_{1}} W_{i, \tau_{1}} I_{k-1, \tau_{1}-}\right\|_{\infty} \leqslant \frac{M^{m}}{J !}\left\|W_{i, \tau_{1}} I_{k-1, \tau_{1}-}\right\|_{\infty} \leqslant \frac{M^{m+1}}{J !}\left\|I_{k-1, \tau_{1}-}\right\|_{\infty}
$$

and so

$$
\left\|A_{J, i, \tau}\right\|_{\infty} \leqslant \frac{1}{2} \frac{d^{-m-1}}{J !} D_{\tau}^{m} \int_{0}^{\tau}\left\|I_{k-1, \tau_{1}-}\right\|_{\infty} d D_{\tau_{1}}
$$


Summing (3.34) over all $J \in \mathcal{B}_{m}, i=1, \ldots, d$, and using the multinomial identity

$$
\sum_{J \in \mathcal{B}_{m}} \frac{1}{J !}=\frac{d^{m}}{m !}
$$

gives (3.30).

To estimate $B_{J, i, \tau}$ we observe that for the continuous BV-processes $W_{j, \tau}$ which satisfy (3.9) one has

$$
d U_{J, \tau_{2}}=\frac{1}{J !} \sum_{r=1}^{d}\left(W_{1, \tau_{2}}\right)^{j_{1}} \ldots\left(d\left(W_{r, \tau_{2}}\right)^{j_{r}}\right) \ldots\left(W_{d, \tau_{2}}\right)^{j_{d}}=\sum_{\substack{r=1 \\ j_{r}>0}}^{d} U_{J-e_{r}, \tau_{2}} d W_{r, \tau_{2}}
$$

where

$$
e_{r}=(\underbrace{0, \ldots, 0,1}_{r}, 0, \ldots, 0) \in \mathbb{N}_{0}^{d} .
$$

Therefore, using (3.5), (3.33) and

$$
\sum_{\substack{r=1 \\ j_{r}>0}}^{d} \frac{1}{\left(J-e_{r}\right) !}=\frac{m}{J !}
$$

we obtain for any càdlàg $\mathcal{F}_{\tau}$-adapted $\mathbb{R}^{M \times M}$-valued process $Z_{\tau}$

$$
\left\|\int_{0}^{\tau} d U_{J, \tau_{2}} \times Z_{\tau_{2}-}\right\|_{\infty} \leqslant \frac{m}{J !} M^{m} \int_{0}^{\tau}\left\|Z_{\tau_{2}-}\right\|_{\infty} d v_{\tau_{2}} .
$$

Note that according to the assumption of the lemma

$$
I_{k, \tau}=\sum_{i=1}^{d} \int_{0}^{\tau-} W_{i, \tau_{1}} I_{k-1, \tau_{1}-} d \eta_{i, \tau_{1}}
$$

(i.e. $\mathcal{M}_{k}=\{1, \ldots, d\}$ in the set $\mathcal{M}=\mathcal{M}_{1} \times \cdots \times \mathcal{M}_{k}$ ) and hence, by the very definition (3.7) of $I_{k, \tau}$

$$
\begin{aligned}
\left\|\sum_{i=1}^{d} B_{J, i, \tau}\right\|_{\infty} & =\left\|\sum_{J^{\prime} \leqslant J} \Re_{J^{\prime}, J, \tau} \int_{0}^{\tau} d U_{J, \tau_{2}} \times \eta_{\tau_{2}}^{J^{\prime}}\left(\sum_{i=1}^{d} \int_{0}^{\tau_{2}-} W_{i, \tau_{1}} I_{k-1, \tau_{1}-} d \eta_{i, \tau_{1}}\right)\right\|_{\infty} \\
& \leqslant \frac{m}{J !} M^{m} \sum_{J^{\prime} \leqslant J}\left|\Re_{J^{\prime}, J, \tau}\right| \int_{0}^{\tau}\left\|\eta_{\tau_{2}-} \sum_{i=1}^{d} \int_{0}^{\tau_{2}-} W_{i, \tau_{1}} I_{k-1, \tau_{1}-} d \eta_{i, \tau_{1}}\right\|_{\infty} d v_{\tau_{2}} \\
& \leqslant \frac{m}{J !} M^{m}\left(\sum_{J^{\prime} \leqslant J}\left|\Re_{J^{\prime}, J, \tau}\right| \sup _{0 \leqslant \tau_{2} \leqslant \tau}\left|\eta_{\tau_{2}}^{J^{\prime}}\right|\right) \int_{0}^{\tau}\left\|I_{k, \tau_{2}-}\right\|_{\infty} d v_{\tau_{2}} \\
& \leqslant \frac{m}{J !} M(2 d)^{-m+1} D_{\tau}^{m-1} \int_{0}^{\tau}\left\|I_{k, \tau_{2}-}\right\|_{\infty} d v_{\tau_{2}},
\end{aligned}
$$


where we used (3.2) of Lemma 3.2. Summing over all $J \in \mathcal{B}_{m}$ and using the multinomial identity (3.35) we get

$$
\left\|\sum_{i=1}^{d} \sum_{J \in \mathcal{B}_{m}} B_{J, i, \tau}\right\|_{\infty} \leqslant \frac{2^{-m+1}}{(m-1) !}(M \cdot d) D_{\tau}^{m-1} \int_{0}^{\tau}\left\|I_{k, \tau_{2}-}\right\|_{\infty} d v_{\tau_{2}} .
$$

Since $\Delta v_{\tau} \leqslant(M \cdot d)^{-1} \Delta D_{\tau}$ we arrive at (3.31).

We proceed with $C_{L, \tau}$. An application of Taylor's formula and (3.25) imply

$$
\left|\delta_{L^{\prime}, \tau_{2}}\right| \leqslant \frac{1}{2}\left(\sup _{0 \leqslant \theta \leqslant 1} \sum_{i, j=1}^{d}\left|\frac{\partial^{2} f_{L^{\prime}}\left(\eta_{\tau_{2}-}+\theta \Delta \eta_{\tau_{2}}\right)}{\partial x_{i} \partial x_{j}}\right|\right) \max _{n=1, \ldots, d}\left|\Delta \eta_{n, \tau_{2}}\right|^{2} .
$$

Since, by $(3.24), f_{L^{\prime}}\left(x_{1}, \ldots, x_{d}\right)=x^{L^{\prime}}=\prod_{j=1}^{d} x_{j}^{l_{j}^{\prime}}$

$$
\sum_{i, j=1}^{d}\left|\frac{\partial^{2} f_{L^{\prime}}(x)}{\partial x_{i} \partial x_{j}}\right| \leqslant\left(m^{\prime}+1\right) m^{\prime} \max _{n=1, \ldots, d}\left|x_{n}\right|^{m^{\prime}-1},
$$

we get from (3.8) and (3.23)

$$
\begin{aligned}
\left|\delta_{L^{\prime}, \tau_{2}}\right| & \leqslant \frac{1}{2}\left(m^{\prime}+1\right) m^{\prime}\left(\max _{i=1, \ldots, d} \sup _{0 \leqslant s \leqslant \tau_{2}}\left(\left|\eta_{i, s}\right|+\left|\Delta \eta_{s}\right|\right)^{m^{\prime}-1}\right)\left(\max _{i=1, \ldots, d}\left|\Delta \eta_{i, \tau_{2}}\right|^{2}\right) \\
& \leqslant \frac{1}{2}\left(m^{\prime}+1\right) m^{\prime}\left((4 M \cdot d)^{-m^{\prime}+1} D_{\tau_{2}}^{m^{\prime}-1}\right)\left((2 M \cdot d)^{-2} D_{\tau_{2}} \Delta D_{\tau_{2}}\right) \\
& \leqslant \frac{1}{2}\left(m^{\prime}+1\right)(2 M \cdot d)^{-m^{\prime}-1} D_{\tau_{2}}^{m^{\prime}} \Delta D_{\tau_{2}} .
\end{aligned}
$$

We apply (3.2) of Lemma 3.2 to find for $L \in \mathcal{B}_{m+1}$

$$
\begin{aligned}
\left\|C_{L, \tau}\right\|_{\infty} & \leqslant \frac{m+1}{2}\left(\sum_{L^{\prime} \leqslant L}\left|\Re_{L^{\prime}, L, \tau}\right|(2 M \cdot d)^{-m^{\prime}-1} D_{\tau}^{m^{\prime}}\right) \int_{0}^{\tau}\left\|U_{L, \tau_{1}} I_{k-1, \tau_{1}-}\right\|_{\infty} d D_{\tau_{1}} \\
& \leqslant \frac{m+1}{2}(M \cdot d)^{-m-1} D_{\tau}^{m} \int_{0}^{\tau}\left\|U_{L, \tau_{1}} I_{k-1, \tau_{1}-}\right\|_{\infty} d D_{\tau_{1}}
\end{aligned}
$$

$$
\leqslant \frac{d^{-m-1}}{2} \frac{(m+1)}{L !} D_{\tau}^{m} \int_{0}^{\tau}\left\|I_{k-1, \tau_{1}-}\right\|_{\infty} d D_{\tau_{1}}
$$

where we used the fact that $\sup _{0 \leqslant s \leqslant t}\left\|W_{j, s}\right\|_{\infty} \leqslant 1$, cf. (3.4). Since

$$
\sum_{L \in \mathcal{B}_{m+1}} \frac{m+1}{L !} d^{-m-1}=\frac{1}{m !}
$$

summing (3.38) over all $L \in \mathcal{B}_{m+1}$ completes the proof. 
Lemma 3.4 For any $m, k \in \mathbb{N}$ one has

$$
\begin{aligned}
& \sum_{i=1}^{d} \sum_{L \in \mathcal{B}_{m+1}} \sum_{L^{\prime} \leqslant L} \mathfrak{R}_{L^{\prime}, L, \tau} \int_{0}^{\tau} l_{i}^{\prime} \eta_{\tau_{2}-}^{L^{\prime}-e_{i}} U_{L, \tau_{2}} I_{k-1, \tau_{2}-} d \eta_{i, \tau_{2}} \\
& =\sum_{i=1}^{d} \sum_{J \in \mathcal{B}_{m}} \sum_{J^{\prime} \leqslant J} \Re_{J^{\prime}, J, \tau} \int_{0}^{\tau} \eta_{\tau_{2}-}^{J^{\prime}} U_{J, \tau_{2}} W_{i, \tau_{2}} I_{k-1, \tau_{2}-} d \eta_{i, \tau_{2}},
\end{aligned}
$$

where $e_{i} \in \mathbb{N}_{0}^{d}$ is the $i$-th unit multi-index.

Proof. Given $L^{\prime}, L \in \mathbb{N}_{0}^{d}, i \in\{1, \ldots, d\}$ such that $L^{\prime} \leqslant L$ and $l_{i}^{\prime}>0$ we take

$$
J=L-e_{i}, \quad J^{\prime}=L^{\prime}-e_{i} .
$$

From (3.19) we find that

$$
\mathfrak{R}_{J^{\prime}, J, \tau}=\frac{l_{i}^{\prime}}{l_{i}} \mathfrak{R}_{L^{\prime}, L, \tau}
$$

Since

$$
U_{J, \tau_{2}} W_{i, \tau_{2}}=\left(j_{i}+1\right) U_{J+e_{i}, \tau_{2}}=l_{i} U_{L, \tau_{2}}
$$

it follows

$$
l_{i}^{\prime} \int_{0}^{\tau} \eta_{\tau_{2}-}^{L^{\prime}-e_{i}} U_{L, \tau_{2}} I_{k-1, \tau_{2}-} d \eta_{i, \tau_{2}}=\frac{l_{i}^{\prime}}{l_{i}} \int_{0}^{\tau} \eta_{\tau_{2}-}^{J^{\prime}} U_{J, \tau_{2}} W_{i, \tau_{2}} I_{k-1, \tau_{2}-} d \eta_{i, \tau_{2}}
$$

Multiplying both sides of (3.42) by $\mathfrak{R}_{L^{\prime}, L, \tau}$ and using (3.41) gives

$$
\begin{aligned}
& \mathfrak{R}_{L^{\prime}, L, \tau} l_{i}^{\prime} \int_{0}^{\tau} \eta_{\tau_{2}-}^{L^{\prime}-e_{i}} U_{L, \tau_{2}} I_{k-1, \tau_{2}-} d \eta_{i, \tau_{2}} \\
&=\mathfrak{R}_{J^{\prime}, J, \tau} \int_{0}^{\tau} \eta_{\tau_{2}-}^{J^{\prime}} U_{J, \tau_{2}} W_{i, \tau_{2}} I_{k-1, \tau_{2}-} d \eta_{i, \tau_{2}} .
\end{aligned}
$$

We sum (3.43) over all triples $\left(L, L^{\prime}, i\right)$ such that $i=1, \ldots, d, L^{\prime} \leqslant L$, $L \in \mathcal{B}_{m+1}$ and $l_{i}^{\prime}>0$. By (3.40), $L=J+e_{i}$ and $L^{\prime}=J^{\prime}+e_{i}$ which means that

$$
\begin{aligned}
\left\{\left(L, L^{\prime}, i\right): i=1, \ldots, d, L^{\prime} \leqslant L \in \mathcal{B}_{m+1}, l_{i}^{\prime}>0\right\} \\
=\left\{\left(J, J^{\prime}, i\right): i=1, \ldots, d, J^{\prime} \leqslant J \in \mathcal{B}_{m}\right\}
\end{aligned}
$$

i.e. we can express the summation of the r.h.s. of (3.43) in terms of $\left(J, J^{\prime}, i\right)$. On the left, the terms where $l_{i}^{\prime}=0$ do not contribute and we may, therefore, simply sum over all $\left(L, L^{\prime}, i\right)$ with $L^{\prime} \leqslant L \in \mathcal{B}_{m+1}, i=1, \ldots, d$. This proves (3.39). 
Using integration by parts for $I_{k, \tau}$ will lead to expressions of the form

$$
Q_{J, i, \tau}=\sum_{J^{\prime} \leqslant J} \Re_{J^{\prime}, J, \tau} \int_{0}^{\tau} U_{J, \tau_{2}}\left(\int_{0}^{\tau_{2}-} W_{i, \tau_{1}} I_{k-1, \tau_{1}-} d \eta_{i, \tau_{1}}\right) d \eta_{\tau_{2}}^{J^{\prime}}
$$

where $J \in \mathbb{N}_{0}^{d}, i \in\{0, \ldots, d\}$ and $U_{J, \tau_{2}}$ is given by (3.26). We want to estimate the norm of the sum $\sum_{|J|=m} Q_{J, i, \tau}$ through the norm of $\sum_{|L|=m+1} Q_{L, i, \tau}$ plus some correction terms. This will be needed in the main induction step in the proof of Proposition 3.1.

Lemma 3.5 For any $m \in \mathbb{N}$ the stochastic integrals $Q_{J, i, \tau}$ satisfy

$$
\begin{aligned}
& \left\|\sum_{i=1}^{d} \sum_{J \in \mathcal{B}_{m}} Q_{J, i, \tau}\right\|_{\infty} \leqslant \\
& \leqslant \frac{D_{\tau}^{m}}{m !} \int_{0}^{\tau}\left\|I_{k-1, \tau_{1}-}\right\|_{\infty} d D_{\tau_{1}}+\frac{D_{\tau}^{m-1}}{(m-1) !} \int_{0}^{\tau}\left\|I_{k, \tau_{2}-}\right\|_{\infty} d D_{\tau_{2}} \\
& \quad+\left\|\sum_{L \in \mathcal{B}_{m+1}} \sum_{L^{\prime} \leqslant L} \Re_{L^{\prime}, L, \tau} \int_{0}^{\tau} U_{L, \tau_{2}} I_{k-1, \tau_{2}-} d \eta_{\tau_{2}}^{L^{\prime}}\right\|_{\infty} .
\end{aligned}
$$

Proof. We write for the right-hand side of (3.45) I + II + III. For any $J \in \mathcal{B}_{m}, i \in\{1, \ldots, d\}$ an application of Corollary 3.1 with $\mathfrak{A}=\left\{J^{\prime} \in \mathbb{N}_{0}\right.$ : $\left.J^{\prime} \leqslant J\right\}, \alpha=J^{\prime}$ and

$$
U_{\tau_{2}}=U_{J, \tau_{2}}, \quad \Phi_{\tau_{1}}=W_{i, \tau_{1}} I_{k-1, \tau_{1}}, \quad \nu_{\tau_{1}}=\eta_{i, \tau_{1}}, \quad \kappa_{\alpha, \tau}=\eta_{\tau}^{J^{\prime}}, \quad \widetilde{\kappa}_{\alpha, \tau}=\Re_{J^{\prime}, J, \tau}
$$

yields

$$
\begin{aligned}
Q_{J, i, \tau}= & -\sum_{J^{\prime} \leqslant J} \mathfrak{R}_{J^{\prime}, J, \tau} \int_{0}^{\tau} U_{J, \tau_{1}} W_{i, \tau_{1}} I_{k-1, \tau_{1}-} d\left[\eta^{J^{\prime}}, \eta_{i}\right]_{\tau_{1}} \\
& -\sum_{J^{\prime} \leqslant J} \mathfrak{R}_{J^{\prime}, J, \tau} \int_{0}^{\tau} d U_{J, \tau_{2}} \times \eta_{\tau_{2}-}^{J^{\prime}} \int_{0}^{\tau_{2}-} W_{i, \tau_{1}} I_{k-1, \tau_{1}-} d \eta_{i, \tau_{1}}, \\
& -\sum_{J^{\prime} \leqslant J} \mathfrak{R}_{J^{\prime}, J, \tau} \int_{0}^{\tau} \eta_{\tau_{2}-}^{J^{\prime}} U_{J, \tau_{2}} W_{i, \tau_{2}} I_{k-1, \tau_{2}-} d \eta_{i, \tau_{2}}
\end{aligned}
$$

where we used that, by (3.20),

$$
\sum_{\alpha \in \mathfrak{A}} \widetilde{\kappa}_{\alpha, \tau} \kappa_{\alpha, \tau}=\sum_{J^{\prime} \leqslant J} \mathfrak{R}_{J^{\prime}, J, \tau} \eta_{\tau}^{J^{\prime}}=0
$$

i.e. (3.17) is satisfied. 
Observe that the first two integrals in (3.46) are just $A_{J, i, \tau}$ and $B_{J, i, \tau}$ defined in (3.27) and (3.28). Therefore, Lemma 3.3 gives

$$
\left\|\sum_{i=1}^{d} \sum_{J \in \mathcal{B}_{m}} Q_{J, i, \tau}\right\|_{\infty} \leqslant \frac{1}{2} \mathbf{I}+\mathbf{I I}+\left\|\sum_{i=1}^{d} \sum_{J \in \mathcal{B}_{m}} \widetilde{C}_{J, i, \tau}\right\|_{\infty},
$$

with I, II as required for $(3.45)$ and with $-\widetilde{C}_{J, i, \tau}$ given by the last term in (3.46). We still have to estimate the last sum.

Recall that $f_{L^{\prime}}\left(x_{1}, \ldots, x_{d}\right)$ and $\delta_{L^{\prime}, \tau_{2}}$ are given by formulae (3.24) and (3.25) respectively. Since the $\eta_{j, \tau}$ are pure-jump semimartingales, an application of Itô's formula to $f_{L^{\prime}}\left(x_{1}, \ldots, x_{d}\right)=x^{L^{\prime}}$ and the process $\eta_{\tau_{2}}=\left(\eta_{1, \tau_{2}}, \ldots, \eta_{d, \tau_{2}}\right)$ yields

$$
\begin{aligned}
\int_{0}^{\tau} U_{L, \tau_{2}} I_{k-1, \tau_{2}-} d \eta_{\tau_{2}}^{L^{\prime}}= & \sum_{i=1}^{d} \int_{0}^{\tau} U_{L, \tau_{2}} I_{k-1, \tau_{2}-} \frac{\partial f_{L^{\prime}}\left(\eta_{\tau_{2}-}\right)}{\partial x_{i}} d \eta_{i, \tau_{2}} \\
& +\sum_{0<\tau_{2} \leqslant \tau} U_{L, \tau_{2}} I_{k-1, \tau_{2}-} \delta_{L^{\prime}, \tau_{2}} .
\end{aligned}
$$

We multiply (3.48) by $\mathfrak{R}_{L^{\prime}, L, \tau}$, sum it over all $L^{\prime} \leqslant L$ and obtain using $\partial f_{L^{\prime}}\left(\eta_{\tau_{2}-}\right) / \partial x_{i}=l_{i}^{\prime} \eta_{\tau_{2}-}^{L^{\prime}-e_{i}}$

$$
\begin{aligned}
& \sum_{L^{\prime} \leqslant L} \Re_{L^{\prime}, L, \tau} \int_{0}^{\tau} U_{L, \tau_{2}} I_{k-1, \tau_{2}-} d \eta_{\tau_{2}}^{L^{\prime}} \\
& \quad=\sum_{i=1}^{d} \sum_{L^{\prime} \leqslant L} \Re_{L^{\prime}, L, \tau} l_{i}^{\prime} \int_{0}^{\tau} \eta_{\tau_{2}-}^{L^{\prime}-e_{i}} U_{L, \tau_{2}} I_{k-1, \tau_{2}-} d \eta_{i, \tau_{2}}+C_{L, \tau}
\end{aligned}
$$

with $C_{L, \tau}$ as in (3.29). Summing (3.49) over all $L \in \mathcal{B}_{m+1}$ and applying Lemma 3.4 we find

$$
\sum_{L \in \mathcal{B}_{m+1}} \sum_{L^{\prime} \leqslant L} \mathfrak{R}_{L^{\prime}, L, \tau} \int_{0}^{\tau} U_{L, \tau_{2}} I_{k-1, \tau_{2}-} d \eta_{\tau_{2}}^{L^{\prime}}=\sum_{i=1}^{d} \sum_{J \in \mathcal{B}_{m}} \widetilde{C}_{J, i, \tau}+\sum_{L \in \mathcal{B}_{m+1}} C_{L, \tau} .
$$

Therefore, by (3.32),

$$
\left\|\sum_{i=1}^{d} \sum_{J \in \mathcal{B}_{m}} \widetilde{C}_{J, i, \tau}\right\|_{\infty} \leqslant\left\|\sum_{L \in \mathcal{B}_{m+1}} C_{L, \tau}\right\|_{\infty}+\mathbf{I I I} \leqslant \frac{1}{2} \mathbf{I}+\mathbf{I I I} .
$$

Combining this and (3.47) we arrive at (3.45). 
Here is the modification of Lemma 3.5 for the case when the inner integral in (3.44) is driven by $d \tau$.

Lemma 3.6 If $\ell_{2 n}<k^{\prime}<\ell_{2 n+1}$ for some $n \in \mathbb{N}$ or $1 \leqslant k^{\prime}<\ell_{1}$ then

$$
\begin{aligned}
& \left\|\sum_{J \in \mathcal{B}_{m}} \sum_{J^{\prime} \leqslant J} \Re_{J^{\prime}, J, \tau} \int_{0}^{\tau} U_{J, \tau_{2}}\left(\int_{0}^{\tau_{2}-} W_{0, \tau_{1}} I_{k^{\prime}-1, \tau_{1}} d \tau_{1}\right) d \eta_{\tau_{2}}^{J^{\prime}}\right\|_{\infty} \\
& \quad \leqslant \frac{M}{m !} D_{\tau}^{m} \int_{0}^{\tau}\left\|I_{k^{\prime}-1, \tau_{2}}\right\|_{\infty} d \tau_{2}+\frac{1}{(m-1) !} D_{\tau}^{m-1} \int_{0}^{\tau}\left\|I_{k^{\prime}, \tau_{2}-}\right\|_{\infty} d D_{\tau_{2}}
\end{aligned}
$$

Proof. For any $J \in \mathcal{B}_{m}, i \in\{1, \ldots, d\}$ we apply Corollary 3.1 with $\mathfrak{A}=$ $\left\{J^{\prime} \in \mathbb{N}_{0}: J^{\prime} \leqslant J\right\}, \alpha=J^{\prime}, \nu_{\tau}=\eta_{0, \tau}=\tau$ and

$$
U_{\tau_{2}}=U_{J, \tau_{2}}, \quad \Phi_{\tau_{1}}=W_{0, \tau_{1}} I_{k^{\prime}-1, \tau_{1}}, \quad \kappa_{\alpha, \tau}=\eta_{\tau}^{J^{\prime}}, \quad \widetilde{\kappa}_{\alpha, \tau}=\mathfrak{R}_{J^{\prime}, J, \tau} .
$$

As before

$$
\sum_{\alpha \in \mathfrak{A}} \widetilde{\kappa}_{\alpha, \tau} \kappa_{\alpha, \tau}=\sum_{J^{\prime} \leqslant J} \mathfrak{R}_{J^{\prime}, J, \tau} \eta_{\tau}^{J^{\prime}}=0 .
$$

Moreover, $\left[\nu, \kappa_{\alpha}\right]=0$. Therefore we estimate the left-hand side of (3.50) by I + II, where

$$
\begin{aligned}
\mathbf{I} & =\left\|\sum_{J \in \mathcal{B}_{m}} \sum_{J^{\prime} \leqslant J} \mathfrak{R}_{J^{\prime}, J, \tau} \int_{0}^{\tau} \eta_{\tau_{2}}^{J^{\prime}} U_{J, \tau_{2}} W_{0, \tau_{2}} I_{k^{\prime}-1, \tau_{2}} d \tau_{2}\right\|_{\infty}, \\
\mathbf{I I} & =\left\|\sum_{J \in \mathcal{B}_{m}} \sum_{J^{\prime} \leqslant J} \mathfrak{R}_{J^{\prime}, J, \tau} \int_{0}^{\tau} d U_{J, \tau_{2}} \times \eta_{\tau_{2}-}^{J^{\prime}} \int_{0}^{\tau_{2}-} W_{0, \tau_{1}} I_{k^{\prime}-1, \tau_{1}} d \tau_{1}\right\|_{\infty} .
\end{aligned}
$$

Similar calculations to those in the proof of Lemma 3.3 give

$$
\begin{aligned}
\mathbf{I} & \leqslant M^{m+1} \sum_{J \in \mathcal{B}_{m}} \frac{1}{J !}\left(\sum_{J^{\prime} \leqslant J}\left|\Re_{J^{\prime}, J, \tau}\right| \sup _{0 \leqslant \tau_{1} \leqslant \tau}\left|\eta_{\tau_{1}}^{J^{\prime}}\right|\right) \int_{0}^{\tau}\left\|I_{k^{\prime}-1, \tau_{2}}\right\|_{\infty} d \tau_{2} \\
& \leqslant M \frac{D_{\tau}^{m}}{m !} \int_{0}^{\tau}\left\|I_{k^{\prime}-1, \tau_{2}}\right\|_{\infty} d \tau_{2} .
\end{aligned}
$$

From (3.37) and arguments similar to those of Lemma 3.3 we deduce

$$
\begin{aligned}
\mathbf{I I} & \leqslant M^{m} \sum_{J \in \mathcal{B}_{m}} \frac{m}{J !}\left(\sum_{J^{\prime} \leqslant J}\left|\Re_{J^{\prime}, J, \tau}\right| \sup _{0 \leqslant \tau_{1} \leqslant \tau}\left|\eta_{\tau_{1}}^{J^{\prime}}\right|\right) \int_{0}^{\tau}\left\|I_{k^{\prime}, \tau_{1}-}\right\|_{\infty} d v_{\tau_{1}} \\
& \leqslant(M d) \frac{D_{\tau}^{m-1}}{(m-1) !} \int_{0}^{\tau}\left\|I_{k^{\prime}, \tau_{1}-}\right\|_{\infty} d v_{\tau_{1}} \\
& \leqslant \frac{D_{\tau}^{m-1}}{(m-1) !} \int_{0}^{\tau}\left\|I_{k^{\prime}, \tau_{1}-}\right\|_{\infty} d D_{\tau_{1}} .
\end{aligned}
$$

Piecing together the above estimates completes the proof. 
Now we are ready to prove Proposition 3.1.

Proof of Proposition 3.1. Without loss of generality we may assume that $t_{0}=0$. We define $p_{l}, l \in \mathbb{N}_{0}$, recursively by $p_{0}=1$ and for

$$
l \in\left(\gamma_{n-1}, \gamma_{n}\right]:=\left(\sum_{i=1}^{n-1}\left(\ell_{2 i}-\ell_{2 i-1}+1\right), \sum_{i=1}^{n}\left(\ell_{2 i}-\ell_{2 i-1}+1\right)\right], \quad \gamma_{0}=0
$$

by

$$
p_{l}=p_{\gamma_{n-1}} q_{l-\gamma_{n-1}},
$$

where the $q_{k}$ are defined by formula (A.3) in the appendix.

Recall that $\mathfrak{m}=\mathfrak{m}(k), D_{\tau}$ were defined at the beginning of this section. Throughout this proof we suppress the argument in $\mathfrak{m}(\cdot)$ if the argument is $k$, i.e. $\mathfrak{m}=\mathfrak{m}(k)$. We split the proof into two steps.

Step 1. We show by induction that

$$
\left\|I_{k, \tau}\right\|_{\infty} \leqslant p_{\mathfrak{m}} D_{\tau}^{\mathfrak{m}} \frac{(M \tau)^{k-\mathfrak{m}}}{(k-\mathfrak{m}) !}\left\|I_{0, \tau}^{*}\right\|_{\infty}, \quad k \in \mathbb{N} .
$$

Clearly, (3.52) is true for $k=0$. Assume that (3.52) holds for $0, \ldots, k-1$.

Case 1. $\ell_{2 n}<k<\ell_{2 n+1}$ for some $n \in \mathbb{N}$ or $1 \leqslant k<\ell_{1}$. In this case $\eta_{j_{k}, \tau}=\tau$ in the definition of $I_{k, \tau}$ and $\mathfrak{m}(k)=\mathfrak{m}(k-1)$. Therefore,

$$
\begin{aligned}
\left\|I_{k, \tau}\right\|_{\infty} \leqslant M \int_{0}^{\tau}\left\|I_{k-1, s}\right\|_{\infty} d s & \leqslant M \int_{0}^{\tau} p_{\mathfrak{m}} D_{s}^{\mathfrak{m}} \frac{(M s)^{k-1-\mathfrak{m}}}{(k-1-\mathfrak{m}) !} d s\left\|I_{0, \tau}^{*}\right\|_{\infty} \\
& \leqslant p_{\mathfrak{m}} D_{\tau}^{\mathfrak{m}} \frac{(M \tau)^{k-\mathfrak{m}}}{(k-\mathfrak{m}) !}\left\|I_{0, \tau}^{*}\right\|_{\infty}
\end{aligned}
$$

which is just (3.52).

Case 2. $\ell_{2 n-1} \leqslant k \leqslant \ell_{2 n}$ for some $n \in \mathbb{N}$; then $\eta_{j_{k}, \tau}$ is a semimartingale. For $\widehat{m} \in \mathbb{N}$ and $\hat{r}=\ell_{2 n-1}, \ldots, k$, we denote by

$$
z_{\hat{r}, \tau}^{\widehat{m}}=\sum_{J \in \mathcal{B}_{\hat{m}}} \sum_{J^{\prime} \leqslant J} \Re_{J^{\prime}, J, \tau} \int_{0}^{\tau} U_{J, s} I_{\hat{r}-1, s-} d \eta_{s}^{J^{\prime}} .
$$

Note that $z_{\hat{r}, \tau}^{1}=I_{\hat{r}, \tau}$ and

$$
z_{\hat{r}+1, \tau}^{\widehat{m}}=\sum_{i=1}^{d} \sum_{J \in \mathcal{B}_{\hat{m}}} Q_{J, i, \tau}, \quad z_{\hat{r}, \tau}^{\widehat{\hat{m}}+1}=\sum_{L \in \mathcal{B}_{\hat{m}+1}} \sum_{L^{\prime} \leqslant L} \Re_{J^{\prime}, J, \tau} \int_{0}^{\tau} U_{L, \tau_{2}} I_{\hat{r}-1, \tau_{2}-} d \eta_{\tau_{2}}^{L^{\prime}},
$$

where

$$
Q_{J, i, \tau}=\sum_{J^{\prime} \leqslant J} \Re_{J^{\prime}, J, \tau} \int_{0}^{\tau} U_{J, \tau_{2}}\left(\int_{0}^{\tau_{2}-} W_{i, \tau_{1}} I_{\hat{r}-1, \tau_{1}-} d \eta_{i, \tau_{1}}\right) d \eta_{\tau_{2}}^{J^{\prime}}
$$


Applying Lemma 3.5 with the above $Q_{J, i, \tau}$ we get

$$
\begin{aligned}
& \left\|z_{\widehat{r}+1, \tau}^{\widehat{m}}\right\|_{\infty} \leqslant \\
& \leqslant \frac{D_{\tau}^{\widehat{m}}}{\widehat{m} !} \int_{0}^{\tau}\left\|I_{\widehat{r}-1, s-}\right\|_{\infty} d D_{s}+\frac{D_{\tau}^{\widehat{m}-1}}{(\widehat{m}-1) !} \int_{0}^{\tau}\left\|I_{\widehat{r}, s-}\right\|_{\infty} d D_{s}+\left\|z_{\widehat{r}, \tau}^{\widehat{m}+1}\right\|_{\infty} .
\end{aligned}
$$

If $k>\ell_{2 n-1}$ we obtain after the change of indices $\widehat{m}=m+1, \widehat{r}+1=k-m$, $m=0,1, \ldots, k-\ell_{2 n-1}-1$,

$$
\left\|z_{k-m, \tau}^{m+1}\right\|_{\infty}-\left\|z_{k-(m+1), \tau}^{m+2}\right\|_{\infty} \leqslant \beta_{m+1, \tau}+\beta_{m, \tau},
$$

where

$$
\beta_{m, \tau}=\frac{D_{\tau}^{m}}{m !} \int_{0}^{\tau}\left\|I_{k-m-1, s}\right\|_{\infty} d D_{s}
$$

Summing (3.54) over $m=0, \ldots,\left(k-\ell_{2 n-1}-1\right)$, we get

$$
\left\|z_{k, \tau}^{1}\right\|_{\infty}-\left\|z_{\ell_{2 n-1}, \tau}^{k-\ell_{2 n-1}+1}\right\|_{\infty} \leqslant \beta_{0, \tau}+\beta_{k-\ell_{2 n-1}, \tau}+2 \sum_{m=1}^{k-\ell_{2 n-1}-1} \beta_{m, \tau} .
$$

Set $k^{\prime}=\ell_{2 n-1}-1$ and observe that $\ell_{2 n-2}<k^{\prime}<\ell_{2 n-1}$. An application of Lemma 3.6 gives

$$
\left\|z_{\ell_{2 n-1}, \tau}^{k-\ell_{2 n-1}+1}\right\|_{\infty} \leqslant \frac{D_{\tau}^{k-\ell_{2 n-1}+1}}{\left(k-\ell_{2 n-1}+1\right) !} M \int_{0}^{\tau}\left\|I_{\ell_{2 n-1}-2, \tau_{2}}\right\|_{\infty} d \tau_{2}+\beta_{k-\ell_{2 n-1}, \tau} .
$$

If $k=\ell_{2 n-1}$, the inequality (3.57) is obviously true by Lemma 3.6. Combining (3.56), (3.57) we arrive at

$$
\left\|z_{k, \tau}^{1}\right\|_{\infty} \leqslant \frac{D_{\tau}^{k-\ell_{2 n-1}+1}}{\left(k-\ell_{2 n-1}+1\right) !} M \int_{0}^{\tau}\left\|I_{\ell_{2 n-1}-2, \tau_{2}}\right\|_{\infty} d \tau_{2}+2 \sum_{m=0}^{k-\ell_{2 n-1}} \beta_{m, \tau} .
$$

Since

$$
\mathfrak{m}(k-m-1)=\mathfrak{m}(k)-m-1, \quad m=0, \ldots, k-\ell_{2 n-1},
$$

we can use the induction hypothesis (3.52) and deduce from (3.55) for $m=$ $0,1, \ldots, k-\ell_{2 n-1}$,

$$
\begin{aligned}
\beta_{m, \tau} & \leqslant \frac{D_{\tau}^{m}}{m !} p_{\mathfrak{m}-m-1} \int_{0}^{\tau} D_{s}^{\mathfrak{m}-m-1} \frac{(M s)^{k-\mathfrak{m}}}{(k-\mathfrak{m}) !} d D_{s}\left\|I_{0, \tau}^{*}\right\|_{\infty} \\
& \leqslant \frac{p_{\mathfrak{m}-m-1}}{(\mathfrak{m}-m) m !} D_{\tau}^{\mathfrak{m}} \frac{(M \tau)^{k-\mathfrak{m}}}{(k-\mathfrak{m}) !}\left\|I_{0, \tau}^{*}\right\|_{\infty} .
\end{aligned}
$$


Since $\mathfrak{m}\left(\ell_{2 n-1}-2\right)=\mathfrak{m}\left(\ell_{2 n-1}-1\right)=\mathfrak{m}(k)-\left(k-\ell_{2 n-1}+1\right)$ we may use again the induction hypothesis $(3.52)$ to get

$$
\begin{aligned}
& M \int_{0}^{\tau}\left\|I_{\ell_{2 n-1}-2, \tau_{2}}\right\|_{\infty} d \tau_{2} \\
& \quad \leqslant p_{\mathfrak{m}-\left(k-\ell_{2 n-1}+1\right)} D_{\tau}^{\mathfrak{m}-\left(k-\ell_{2 n-1}+1\right)} M \int_{0}^{\tau} \frac{(M s)^{k-\mathfrak{m}-1}}{(k-\mathfrak{m}-1) !} d s\left\|I_{0, \tau}^{*}\right\|_{\infty} .
\end{aligned}
$$

Together (3.58), (3.60) and (3.61) show

$$
\begin{aligned}
\left\|z_{k, \tau}^{1}\right\|_{\infty} \leqslant & p_{\mathfrak{m}-\left(k-\ell_{2 n-1}+1\right)} \frac{D_{\tau}^{\mathfrak{m}}}{\left(k-\ell_{2 n-1}+1\right) !} \frac{(M \tau)^{k-\mathfrak{m}}}{(k-\mathfrak{m}) !}\left\|I_{0, \tau}^{*}\right\|_{\infty} \\
& +2 \sum_{m=0}^{k-\ell_{2 n-1}} \frac{p_{\mathfrak{m}-m-1} D_{\tau}^{\mathfrak{m}}}{(\mathfrak{m}-m) m !} \frac{(M \tau)^{k-\mathfrak{m}}}{(k-\mathfrak{m}) !}\left\|I_{0, \tau}^{*}\right\|_{\infty} .
\end{aligned}
$$

Since $\mathfrak{m}-\left(k-\ell_{2 n-1}+1\right)=\gamma_{n-1}$ it follows from (3.51), (3.59) with $l=\mathfrak{m}-m-1$ that

$$
p_{\mathfrak{m}-m-1}=p_{\gamma_{n-1}} q_{\left(k-\ell_{2 n-1}+1\right)-m-1}, \quad m=0, \ldots, k-\ell_{2 n-1} .
$$

From this and definition (A.3) in the appendix we find

$$
\begin{aligned}
\frac{p_{\mathfrak{m}-\left(k-\ell_{2 n-1}+1\right)}}{\left(k-\ell_{2 n-1}+1\right) !}+\sum_{m=0}^{k-\ell_{2 n-1}} \frac{2 p_{\mathfrak{m}-m-1}}{(\mathfrak{m}-m) m !} & \leqslant p_{\gamma_{n-1}} \sum_{m=0}^{k-\ell_{2 n-1}} \frac{3 q_{\left(k-\ell_{2 n-1}+1\right)-m-1}}{\left(\left(k-\ell_{2 n-1}+1\right)-m\right) m !} \\
& =p_{\gamma_{n-1}} q_{k-\ell_{2 n-1}+1}=p_{\mathfrak{m}},
\end{aligned}
$$

where we used $\mathfrak{m} \geqslant k-\ell_{2 n-1}+1$. Combining (3.62) and (3.63) we arrive at (3.52).

Step 2. We are going to prove that

$$
\frac{p_{\mathfrak{m}}}{(k-\mathfrak{m}) !} \leqslant \frac{\left(2^{7}\right)^{k}}{(\ln \ln (k+1))^{\frac{k}{16}}} .
$$

If $1 \leqslant k<\ell_{1}$ then $\mathfrak{m}(k)=0, p_{\mathfrak{m}}=p_{0}=1$, and estimate (3.64) is clear. From definition (3.51) we deduce

$$
p_{\mathfrak{m}}=q_{k-\ell_{2 n-1}+1} \prod_{j=1}^{n-1} q_{\ell_{2 j}-\ell_{2 j-1}+1} \quad \text { for } \quad \ell_{2 n-1} \leqslant k \leqslant \ell_{2 n}
$$

and

$$
p_{\mathfrak{m}}=\prod_{j=1}^{n} q_{\ell_{2 j}-\ell_{2 j-1}+1} \quad \text { for } \quad \ell_{2 n}<k<\ell_{2 n+1} .
$$


From (A.4) we know

$$
\frac{p_{\mathfrak{m}}}{(k-\mathfrak{m}) !} \leqslant\left(2^{6}\right)^{k} Z^{-1}
$$

where

$$
Z=(k-\mathfrak{m}) ! \prod_{j=1}^{n}\left(\ln \left\{\alpha_{j}+1\right\}\right)^{\frac{\alpha_{j}}{2}} .
$$

Here $\alpha_{j}=\ell_{2 j}-\ell_{2 j-1}+1, \quad j=1, \ldots, n-1$, and $\alpha_{n}=k-\ell_{2 n-1}+1$ for $\ell_{2 n-1} \leqslant k \leqslant \ell_{2 n}, \alpha_{n}=\ell_{2 n}-\ell_{2 n-1}+1$ for $\ell_{2 n}<k<\ell_{2 n+1}$. Clearly $k-\mathfrak{m} \geqslant n-1$. Using the estimate from Lemma $A .3$ with $m=k-\mathfrak{m}$ gives

$$
Z \geqslant \frac{1}{2^{k}}(\ln \ln (k+1))^{\frac{k}{16}}
$$

and (3.65), (3.66) show (3.64). The proposition now follows from (3.52) and (3.64).

\section{Boundary value problems for stochastic Hamilton systems (diffeomorphism theorem)}

We consider the following Hamiltonian system

$$
\left\{\begin{array}{l}
d x=p d t \\
d p=\frac{\partial V(x)}{\partial x} d t-\frac{\partial c(x)}{\partial x} d \xi_{t}
\end{array}\right.
$$

with initial condition $\left(x_{0}, p_{0}\right) \in \mathbb{R}^{2 d}$ at $t=t_{0}$. We write

$$
(X, P)=\left(X\left(t, t_{0}, x_{0}, p_{0}\right), P\left(t, t_{0}, x_{0}, p_{0}\right)\right) \in \mathbb{R}^{2 d}
$$

for its solution. The coefficients

$$
\frac{\partial V}{\partial x} \in \mathbb{R}^{d}, \quad \frac{\partial c}{\partial x} \in \mathbb{R}^{d \times d}
$$

are derivatives of functions $V: \mathbb{R}^{d} \rightarrow \mathbb{R}^{1}$ and $c=\left(c_{1}, \ldots, c_{d}\right): \mathbb{R}^{d} \rightarrow \mathbb{R}^{d}$ which admit (at least) continuous partial derivatives up to order 3 such that

$$
\left|\frac{\partial^{|L|} V(x)}{\partial x^{L}}\right|,\left|\frac{\partial^{|L|} c(x)}{\partial x^{L}}\right| \leqslant K \quad|L|=2,3
$$

and

$$
\frac{\partial c(x)}{\partial x}=0, \quad \forall \quad|x|>K
$$

for some constant $K>1$. 
The driving noise $\xi_{t}=\left(\xi_{1, t}, \ldots, \xi_{d, t}\right)$ is a $d$-dimensional Lévy process such that

$$
\left[\xi_{j}, \xi_{j}\right]^{c}=0, \quad j=1, \ldots, d,
$$

i.e. it contains no Brownian component. The main result of this section is the following.

Theorem 4.1 Under the assumptions (4.2)-(4.4), there exists a stopping time $T$ such that $\mathbb{P}(T>0)=1$ and for $0 \leqslant t_{0}<t<T(\omega), x_{0} \in \mathbb{R}^{d}$,

(i) the system (4.1) has a solution $(X, P)$,

$$
\begin{aligned}
& \frac{\partial X}{\partial x_{0}}=E_{d}+O\left(t-t_{0}\right), \quad \frac{\partial P}{\partial p_{0}}=E_{d}+O\left(t-t_{0}\right), \\
& \frac{\partial X}{\partial p_{0}}=\left(t-t_{0}\right) E_{d}+O\left(\left(t-t_{0}\right)^{2}\right),
\end{aligned}
$$

where $O(\cdot)$ is uniform with respect to $x_{0}, p_{0}$,

(ii) the map

$$
\mathfrak{D}: \mathbb{R}^{d} \rightarrow \mathbb{R}^{d}, \quad p_{0} \mapsto X\left(t, t_{0}, x_{0}, p_{0}\right)
$$

is a diffeomorphism.

Remark. We can rewrite the system (4.1) in the following form

$$
\left(\begin{array}{c}
X(t) \\
P(t)
\end{array}\right)=\int_{t_{0}}^{t} \mathcal{V}(X(s), P(s)) d s-\int_{t_{0}}^{t} \gamma(X(s), P(s)) d \zeta_{s}
$$

with coefficients

$$
\mathcal{V}(x, p)=\left(\begin{array}{c}
p \\
\partial V(x) / \partial x
\end{array}\right) \in \mathbb{R}^{2 d}, \quad \gamma(x, p)=\left(\begin{array}{cc}
0 & 0 \\
0 & \partial c(x) / \partial x
\end{array}\right) \in \mathbb{R}^{2 d \times 2 d}
$$

and the (degenerate) Lévy noise

$$
\zeta_{t}=\left(\begin{array}{c}
0 \\
\xi_{t}
\end{array}\right) \in \mathbb{R}^{2 d}
$$

Notice that $\mathcal{V}$ and $\gamma$ are globally Lipschitz continuous; Theorem 7 of [Pro, pp. 197-198] guarantees existence and uniqueness of a solution

$$
\left\{\left(\begin{array}{l}
X(t) \\
P(t)
\end{array}\right)\right\}_{t \geqslant t_{0}}
$$

Moreover, if the coefficients have globally Lipschitz continuous partial derivatives up to order $N+2$, then we may differentiate

$$
\left\{\left(\begin{array}{l}
X(t) \\
P(t)
\end{array}\right)\right\}_{t \geqslant t_{0}}
$$

w.r.t. the initial conditions up to order $N$, cf. [Pro, p. 254, Theorem 40]. 
For the proof of Theorem 4.1 we need the following auxiliary result.

Lemma 4.1 There exists a constant $K_{1}=K_{1}(K, d)$ such that for $t_{0} \leqslant a \leqslant$ $b<\mathcal{R} \wedge K_{1}^{-1}$ (R is the stopping time from Lemma 2.1 and $K$ is the constant from $(4.2))$

$$
\int_{a}^{b}|P(\tau)| d \tau \leqslant 3|X(a)-X(b)|+K_{1}(b-a)
$$

where

$$
X(\tau)=X\left(\tau, t_{0}, x_{0}, p_{0}\right), \quad P(\tau)=P\left(\tau, t_{0}, x_{0}, p_{0}\right) .
$$

Proof. Step 1. From the system (4.1) we find

$$
P(\tau)-P(a)=\int_{a}^{\tau} \frac{\partial V(X(s))}{\partial x} d s-\int_{a}^{\tau} \frac{\partial c(X(s))}{\partial x} d \xi_{s} .
$$

Since $X(t), \partial V(X(t)) / \partial x$ and $\partial c(X(t)) / \partial x$ are continuous BV-processes, we find by integration by parts

$$
\begin{aligned}
P(\tau) & -P(a)=\frac{\partial V(X(a))}{\partial x}(\tau-a)-\frac{\partial c(X(a))}{\partial x}\left(\xi_{\tau}-\xi_{a}\right) \\
& -\int_{a}^{\tau}(s-\tau) \frac{\partial^{2} V(X(s))}{\partial x^{2}} P(s) d s+\int_{a}^{\tau} \frac{\partial^{2} c(X(s))\left(\xi_{s}-\xi_{a}\right)}{\partial x^{2}} P(s) d s
\end{aligned}
$$

Here

$$
\frac{\partial^{2} c(X(s))\left(\xi_{s}-\xi_{a}\right)}{\partial x^{2}}=\sum_{i=1}^{d} \frac{\partial^{2} c_{i}(X(s))\left(\xi_{i, s}-\xi_{i, a}\right)}{\partial x^{2}} \in \mathbb{R}^{d \times d} .
$$

We know from Lemma 2.1 that

$$
\left|\xi_{\tau}-\xi_{a}\right| \leqslant 2 \sup _{0 \leqslant s \leqslant \tau}\left|\xi_{s}\right| \leqslant 2 \sum_{i=1}^{d} \sup _{0 \leqslant s \leqslant \tau}\left|\xi_{i, s}\right| \leqslant \vartheta_{t}<1
$$

for $\tau<\mathcal{R}$ and so

$$
|P(\tau)| \leqslant|P(a)|+C_{1}+C_{1} \int_{a}^{\tau}|P(s)| d s
$$

where

$$
C_{1}=\max _{i=1, \ldots, d} \sup _{x \in \mathbb{R}^{d}}\left(d\left\|\frac{\partial^{2} V}{\partial x^{2}}\right\|_{\infty}+d\left\|\frac{\partial^{2} c_{i}}{\partial x^{2}}\right\|_{\infty}\right) \vee\left(\sqrt{d}\left\|\frac{\partial V}{\partial x}\right\|_{\infty}+\sqrt{d}\left\|\frac{\partial c}{\partial x}\right\|_{\infty}\right) .
$$

Integrating (4.9) we have for $b<\mathcal{R}$

$$
\int_{a}^{b}|P(\tau)| d \tau \leqslant|P(a)|(b-a)+C_{1}(b-a)+C_{1} \int_{a}^{b} \int_{a}^{\tau}|P(s)| d s d \tau .
$$


Since for $b-a<\left(3 C_{1}\right)^{-1}$

$$
\begin{aligned}
C_{1} \int_{a}^{b} \int_{a}^{\tau}|P(s)| d s d \tau & =C_{1}(b-a) \int_{a}^{b}|P(s)| d s-C_{1} \int_{a}^{b}(\tau-a)|P(\tau)| d \tau \\
& \leqslant \frac{1}{3} \int_{a}^{b}|P(s)| d s
\end{aligned}
$$

we deduce from (4.10) that

$$
\int_{a}^{b}|P(\tau)| d \tau \leqslant \frac{3}{2}\left(|P(a)|+C_{1}\right)(b-a) .
$$

Step 2. Similarly, we find from (4.8)

$$
|P(\tau)-P(a)| \leqslant C_{1}+C_{1} \int_{a}^{\tau}|P(s)| d s .
$$

We integrate (4.13) to get

$$
\begin{aligned}
\int_{a}^{b}|P(\tau)-P(a)| d \tau & \leqslant C_{1}(b-a)+C_{1} \int_{a}^{b} \int_{a}^{\tau}|P(s)| d s d \tau \\
& \leqslant C_{1}(b-a)+\frac{1}{3} \int_{a}^{b}|P(s)| d s
\end{aligned}
$$

where we used (4.11) and so, by (4.12)

$$
\int_{a}^{b}|P(\tau)-P(a)| d \tau \leqslant \frac{1}{2}\left(|P(a)|+3 C_{1}\right)(b-a) .
$$

Thus

$$
\begin{aligned}
\left|\int_{a}^{b} P(\tau) d \tau\right| & \geqslant|P(a)|(b-a)-\int_{a}^{b}|P(\tau)-P(a)| d \tau \\
& \geqslant \frac{1}{2}\left(|P(a)|-3 C_{1}\right)(b-a) .
\end{aligned}
$$

Combining (4.12) and (4.14) we arrive at

$$
\int_{a}^{b}|P(\tau)| d \tau \leqslant 6 C_{1}(b-a)+3\left|\int_{a}^{b} P(\tau) d \tau\right| .
$$

The assertion follows with $K_{1}=6 C_{1}$. 
Corollary 4.1 Let $f: \mathbb{R}^{d} \rightarrow \mathbb{R}, f \in C^{1}$ and

$$
f^{(1)}(x)=0 \quad \text { if } \quad|x|>K
$$

( $K$ is the constant from (4.2)). Then for $0 \leqslant t_{0} \leqslant t<\mathcal{R} \wedge K_{2}^{-1}$

$$
\operatorname{Var}_{\left[t_{0}, t\right]} f(X(\cdot)) \leqslant K_{2}
$$

where $K_{2}=K_{2}(K, d, f)$ is some constant.

Proof. Let $\mathcal{B}=\left\{\tau \in\left[t_{0}, t\right]:|X(\tau)| \leqslant K\right\}$. If $\mathcal{B}=\emptyset$ then the left-hand side of (4.15) vanishes and the assertion of the corollary is clear. Otherwise we set $a=\inf \{\tau: \tau \in \mathcal{B}\}, b=\sup \{\tau: \tau \in \mathcal{B}\}$.

Since the first derivative $f^{(1)}$ has compact support, supp $f^{(1)} \subset\{x:|x| \leqslant K\}$,

$$
\begin{aligned}
\operatorname{Var}_{\left[t_{0}, t\right]} f(X(\cdot)) & =\int_{a}^{b}\left|f^{(1)}(X(s)) P(s)\right| d s \\
& \leqslant \sup _{x \in \mathbb{R}^{d}}\left|f^{(1)}(x)\right| \int_{a}^{b}|P(\tau)| d \tau
\end{aligned}
$$

and (4.15) follows from Lemma 4.1.

We introduce a new stopping time

$$
T=\mathcal{R} \wedge \widetilde{K}_{2}^{-1}
$$

where $\widetilde{K}_{2}=\max _{i, j=1, \ldots, d} K_{2}\left(K, d, \partial c_{i} / \partial x_{j}\right)$ and $K_{2}$ is defined in Corollary 4.1.

Proof of Theorem 4.1. Step 1. Since $(X, P)$ can be differentiated with respect to the initial data $\left(x_{0}, p_{0}\right)$, we find that the matrix-valued process

$$
G=\frac{\partial(X, P)}{\partial\left(x_{0}, p_{0}\right)}=\left(\begin{array}{cc}
\partial X / \partial x_{0} & \partial X / \partial p_{0} \\
\partial P / \partial x_{0} & \partial P / \partial p_{0}
\end{array}\right)
$$

satisfies the formally differentiated system (4.1) (cf. also [Pro], proof of Theorem 39, p. 250):

$$
d G=W_{0, t} G d t+\sum_{j=1}^{d} W_{j, t} G d \xi_{j, t},\left.\quad G\right|_{t=t_{0}}=G_{0}=\left(\begin{array}{cc}
E_{d} & 0 \\
0 & E_{d}
\end{array}\right)
$$

where $E_{d} \in \mathbb{R}^{d \times d}$ is the identity matrix and

$$
W_{0, t}=\left(\begin{array}{cc}
0 & E_{d} \\
\partial^{2} V(X(t)) / \partial x^{2} & 0
\end{array}\right), W_{j, t}=\left(\begin{array}{cc}
0 & 0 \\
-\partial^{2} c_{j}(X(t)) / \partial x^{2} & 0
\end{array}\right) .
$$


A solution of the system (4.16) can be given by the following (formal) series expansion,

$$
G=\sum_{k=0}^{\infty} G_{k}
$$

with $G_{0}=\left.G\right|_{t=t_{0}}$

$G_{1}=\sum_{j=0}^{d} \int_{t_{0}}^{t} W_{j, \tau} G_{0} d \eta_{j, \tau} \quad$ and $\quad G_{k}=\sum_{j=0}^{d} \int_{t_{0}}^{t} W_{j, \tau} G_{k-1} d \eta_{j, \tau}, \quad(k \in \mathbb{N})$

where $\eta_{\tau}=\left(\eta_{0, \tau}, \eta_{1, \tau}, \ldots, \eta_{d, \tau}\right)=\left(\tau, \xi_{1, \tau}, \ldots, \xi_{d, \tau}\right)$ is a $(d+1)$-dimensional semimartingale.

Indeed, it is immediate that

$$
\sum_{j=0}^{d} \int_{t_{0}}^{t} W_{j, \tau}\left(G_{0}+G_{1}+\cdots+G_{k}\right) d \eta_{j, \tau}=G_{1}+\cdots+G_{k+1},
$$

so (4.18) will give a solution of (4.16) whenever it converges uniformly (on compact intervals) in $t$.

Since the terms of series (4.18) are $k$-fold integrals, we get

$$
G=E_{2 d}+\sum_{k=1}^{\infty} \sum_{j_{1}, \ldots, j_{k}=0}^{d} \widetilde{I}_{j_{1}, \ldots, j_{k}, t}=E_{2 d}+\widetilde{I}_{0, t}+\left(\begin{array}{cc}
A_{11} & A_{12} \\
A_{21} & A_{22}
\end{array}\right),
$$

where $A_{i j} \in \mathbb{R}^{d \times d}$ are suitable (series of) block-matrices and

$$
\widetilde{I}_{j_{1}, \ldots, j_{k}, t}=\int_{t_{0}}^{t} W_{j_{k}, \tau_{k}}\left(\int_{t_{0}}^{\tau_{k}-} \ldots\left(\int_{t_{0}}^{\tau_{2}-} W_{j_{1}, \tau_{1}} d \eta_{j_{1}, \tau_{1}}\right) \ldots d \eta_{j_{k-1}, \tau_{k-1}}\right) d \eta_{j_{k}, \tau_{k}}
$$

Because of the particular form of the $W_{j, \tau}$ 's in (4.17), we know more about the structure of $A_{i j}$ in (4.19). Let

$$
\mathcal{J}_{1}=\left\{\left(j_{1}, \ldots, j_{k}\right): k \in \mathbb{N} \text {, none of } j_{1}, \ldots, j_{k} \text { equals to } 0\right\}
$$

(i.e. all integrators in (4.20) are Lévy processes) and

$$
\mathcal{J}_{2}=\left\{\left(j_{1}, \ldots, j_{k}\right): k \geqslant 2 \text { at most one } j_{1}, \ldots, j_{k} \text { equals to } 0\right\}
$$

(i.e. at most one $d \tau$ integration happens). If $\left(j_{1}, \ldots, j_{k}\right) \in \mathcal{J}_{1}$, then the iterated integrals have the form

$$
\widetilde{I}_{j_{1}, \ldots, j_{k}, t}=\left(\begin{array}{ll}
0 & 0 \\
r & 0
\end{array}\right), \quad r \in \mathbb{R}^{d \times d}
$$

and if $\left(j_{1}, \ldots, j_{k}\right) \in \mathcal{J}_{2}$, they are of the form

$$
\widetilde{I}_{j_{1}, \ldots, j_{k}, t}=\left(\begin{array}{cc}
r_{11} & 0 \\
r_{21} & r_{22}
\end{array}\right), \quad r_{11}, r_{21}, r_{22} \in \mathbb{R}^{d \times d} .
$$


Thus

$$
\begin{aligned}
\left\|A_{11}\right\|_{\infty},\left\|A_{22}\right\|_{\infty} & \leqslant\left\|\sum_{\left(j_{1}, \ldots, j_{k}\right) \in \mathcal{J} \backslash \mathcal{J}_{1}} \widetilde{I}_{j_{1}, \ldots, j_{k}, t}\right\|_{\infty}, \\
\left\|A_{12}\right\|_{\infty} & \leqslant\left\|\sum_{\left(j_{1}, \ldots, j_{k}\right) \in \mathcal{J} \backslash \mathcal{J}_{2}} \widetilde{I}_{j_{1}, \ldots, j_{k}, t}\right\|_{\infty},
\end{aligned}
$$

where $\mathcal{J}=\cup_{k=1}^{\infty}\{0, \ldots, d\}^{k}$.

Step 2. Let us now verify the conditions needed in Proposition 3.1. Lemma 2.1 and condition (4.4) imply that $\eta_{t}=\left(t, \xi_{t}\right)$ satisfies (3.6) for $0 \leqslant t_{0} \leqslant t<\mathcal{R}$.

Condition (4.2) implies that for some constant $K>1$

$$
\left\|W_{j, t}\right\|_{\infty} \leqslant K, \quad j=0, \ldots, d
$$

and, by (4.17), we find

$$
W_{j, \tau} W_{i, \tau}=0=W_{i, \tau} W_{j, \tau} \quad i, j=1, \ldots, d .
$$

Definition (3.8) (with $\mathrm{M}=2 \mathrm{~d}$ ) and formula (4.15) with $f=\left(\partial c_{i} / \partial x_{j}\right)$, $i, j=1, \ldots, d$, give

$$
D_{t} \leqslant 2 d^{2}\left(\widetilde{K}_{2}+2 \vartheta_{t}+2 \vartheta_{t_{0}}\right) \leqslant 2 d^{2}\left(\widetilde{K}_{2}+4 \vartheta_{t}\right),
$$

where $\vartheta_{t}, \vartheta_{t_{0}}$ are given by formula (2.4), and Lemma 2.1 shows

$$
D_{t} \leqslant 2 d^{2}\left(\widetilde{K}_{2}+4\right)=O(1), \quad 0 \leqslant t_{0} \leqslant t<T .
$$

Step 3. For any

$$
\mathcal{M}^{k}=\mathcal{M}_{1}^{k} \times \cdots \times \mathcal{M}_{k}^{k}, \quad \mathcal{M}_{i}^{k}=\{1, \ldots, d\} \quad \text { or } \quad \mathcal{M}_{i}^{k}=\{0\}
$$

an application of Proposition 3.1 to the matrices $K^{-1} W_{j, \tau} \in \mathbb{R}^{2 d \times 2 d}, \quad j=$ $1, \ldots, d$, shows (note that $I_{0, \tau}=E_{2 d}$ is the identity!)

$$
\left\|\sum_{\left(j_{1}, \ldots, j_{k}\right) \in \mathcal{M}^{k}} \widetilde{I}_{j_{1}, \ldots, j_{k}, t}\right\|_{\infty} \leqslant K^{k} b_{k} D_{t}^{\mathfrak{m}}\left\{2 d\left(t-t_{0}\right)\right\}^{k-\mathfrak{m}},
$$

where $b_{k}$ are given by (3.11). One readily sees

$$
\text { (4.25) } \mathcal{M}^{k} \cap \mathcal{J}_{1}=\emptyset \Longrightarrow \mathfrak{m} \leqslant k-1 \text { and } \mathcal{M}^{k} \cap \mathcal{J}_{2}=\emptyset \Longrightarrow \mathfrak{m} \leqslant k-2 \text {. }
$$

Consequently, using the fact that the set $\{0, \ldots, d\}^{k}$ is the disjoint union of $2^{k}$ subsets of type (4.24), we find from (4.21)

$$
\left\|A_{11}\right\|_{\infty},\left\|A_{22}\right\|_{\infty} \leqslant \sum_{k=2}^{\infty} 2^{k} \max \left\|\sum_{\left(j_{1}, \ldots, j_{k}\right) \in \mathcal{M}^{k}} \widetilde{I}_{j_{1}, \ldots, j_{k}, t}\right\|_{\infty},
$$

where the maximum is taken over all $\mathcal{M}^{k}$ such that $\mathfrak{m} \leqslant k-1$. 
Hence

$$
\left\|A_{11}\right\|_{\infty},\left\|A_{22}\right\|_{\infty} \leqslant \sum_{k=2}^{\infty} 2^{k} K^{k} b_{k}\left(D_{t}+2 d\left(t-t_{0}\right)\right)^{k-1}(2 d)\left(t-t_{0}\right),
$$

where we used that, by $(4.25), \mathfrak{m} \leqslant k-1$, and so

$$
D_{t}^{\mathfrak{m}}\left\{2 d\left(t-t_{0}\right)\right\}^{k-\mathfrak{m}} \leqslant\left(D_{t}+2 d\left(t-t_{0}\right)\right)^{k-1}(2 d)\left(t-t_{0}\right) .
$$

Since the coefficients $b_{k}$ from (3.11) are rapidly decreasing, it is clear that the series $\Phi_{1}(x)=\sum_{k=2}^{\infty} 2^{k} K^{k} b_{k} x^{k-1}$ converges for all $x \in \mathbb{R}$; by (4.23), $\Phi_{1}\left(D_{t}+2 d\left(t-t_{0}\right)\right)=O(1)$. Then we deduce from (4.26) that

$$
\left\|A_{11}\right\|_{\infty},\left\|A_{22}\right\|_{\infty}=O\left(t-t_{0}\right), \quad 0 \leqslant t_{0} \leqslant t<T .
$$

Similarly, using (4.22) and (4.25) we have

$$
\begin{aligned}
\left\|A_{12}\right\|_{\infty} & \leqslant \sum_{k=2}^{\infty} 2^{k} K^{k} b_{k}\left(D_{t}+2 d\left(t-t_{0}\right)\right)^{k-2}(2 d)^{2}\left(t-t_{0}\right)^{2} \\
& \leqslant(2 d)^{2} \Phi_{2}\left(D_{t}+2 d\left(t-t_{0}\right)\right)\left(t-t_{0}\right)^{2} \\
& =O\left(\left(t-t_{0}\right)^{2}\right),
\end{aligned}
$$

where $\Phi_{2}(x)=\sum_{k=2}^{\infty} 2^{k} K^{k} b_{k} x^{k-2}$. Substituting estimates (4.27), (4.28) into (4.19) we arrive at (4.5), (4.6).

Step 4. From (4.6), we conclude (using the implicit function theorem) that the map $\mathfrak{D}: p_{0} \mapsto X\left(t, p_{0}\right)=X\left(t, t_{0}, x_{0}, p_{0}\right)$ is a local diffeomorphism. Let us prove that it is injective. Since

$$
X\left(t, p_{2}\right)-X\left(t, p_{1}\right)=\int_{0}^{1} \frac{\partial X}{\partial p_{0}}\left(t, p_{1}+\tau\left(p_{2}-p_{1}\right)\right)\left(p_{2}-p_{1}\right) d \tau,
$$

we have

$$
\begin{aligned}
\left|X\left(t, p_{2}\right)-X\left(t, p_{1}\right)\right|^{2}= & \int_{0}^{1} \int_{0}^{1}\left(p_{2}-p_{1}\right)^{T}\left(\frac{\partial X}{\partial p_{0}}\left(t, p_{1}+s\left(p_{2}-p_{1}\right)\right)\right)^{T} \times \\
& \times\left(\frac{\partial X}{\partial p_{0}}\left(t, p_{1}+\tau\left(p_{2}-p_{1}\right)\right)\right)\left(p_{2}-p_{1}\right) d \tau d s \\
\geqslant & C\left\|p_{2}-p_{1}\right\|^{2},
\end{aligned}
$$

for some constant $C=C\left(t, t_{0}\right)>0$. The last inequality comes from (4.6). This shows that $\mathfrak{D}$ is injective and so $\mathfrak{D}: \mathbb{R}^{d} \rightarrow \mathfrak{D}\left(\mathbb{R}^{d}\right) \subset \mathbb{R}^{d}$ is a global diffeomorphism. Since, by (4.29), $\mathfrak{D}$ is open and closed, then $\mathfrak{D}\left(\mathbb{R}^{d}\right) \subset \mathbb{R}^{d}$ is open and closed. As $\mathfrak{D}\left(\mathbb{R}^{d}\right) \neq \emptyset$ we have $\mathfrak{D}\left(\mathbb{R}^{d}\right)=\mathbb{R}^{d}$. This finishes the proof of Theorem 4.1 . 
We assume that for all multi-indices $I \in \mathbb{N}_{0}^{d}, 2 \leqslant|I| \leqslant q+2$ the partial derivatives

$$
\left|\frac{\partial^{|I|} V}{\partial x^{I}}\right| \leqslant K, \quad\left|\frac{\partial^{|I|} c_{i}}{\partial x^{I}}\right| \leqslant K, \quad i=1, \ldots, d,
$$

are bounded and continuous.

Lemma 4.2 If the coefficients $V, c_{i}$ satisfy the above mentioned assumptions, we have for all $0 \leqslant t_{0} \leqslant t<T$

$$
\begin{aligned}
& \frac{\partial^{|I|} X\left(t, t_{0}, x_{0}, p_{0}\right)}{\partial p_{0}^{I}}=O\left(\left(t-t_{0}\right)^{|I|+1}\right), \\
& \frac{\partial^{|I|} P\left(t, t_{0}, x_{0}, p_{0}\right)}{\partial p_{0}^{I}}=O\left(\left(t-t_{0}\right)^{|I|}\right),
\end{aligned}
$$

where $I \in \mathbb{N}_{0}^{d}, 2 \leqslant|I| \leqslant q$ and $O(\cdot)$ is uniform with respect to $x_{0}$ and $p_{0}$.

For notational convenience we set $c_{0}(X)=-V(X)$. Let us first prove a technical lemma.

Lemma 4.3 For $r \in \mathbb{N}, i_{1}, \ldots, i_{r} \in\{1, \ldots, d\}, i \in\{0 \ldots, d\}$ and $\rho, \mu, \ldots$, $\lambda, L \in \mathbb{N}_{0}^{d},|\rho|,|\mu|, \ldots,|L| \leqslant q$, we define $Z_{i}(\tau)=Z_{i, i_{1}, \ldots, i_{r}, \rho, \mu, \ldots, \lambda, L}(\tau)$ by

$$
Z_{i}(\tau)=\frac{\partial^{|\mu|} X_{i_{1}}(\tau)}{\partial p_{0}^{\mu}} \ldots \frac{\partial^{|\lambda|} X_{i_{r}}(\tau)}{\partial p_{0}^{\lambda}} \frac{\partial^{|\rho|} c_{i}^{(2)}(X(\tau))}{\partial x^{\rho}}\left(\frac{\partial^{|L|}}{\partial p_{0}^{L}} \frac{\partial X}{\partial p_{0}}\right),
$$

where $c_{i}^{(2)}(x)=\left(\partial^{2} c_{i}(x) / \partial x^{2}\right) \in \mathbb{R}^{d \times d}$. Then

$$
\begin{aligned}
& \left\|\sum_{i=0}^{d} \int_{t_{0}}^{s} Z_{i}(\tau) d \eta_{i, \tau}\right\|_{\infty} \\
& \leqslant C \int_{t_{0}}^{s}\left|\frac{\partial^{|\mu|} P(\tau)}{\partial p_{0}^{\mu}}\right| d \tau \ldots \int_{t_{0}}^{s}\left|\frac{\partial^{|\lambda|} P(\tau)}{\partial p_{0}^{\lambda}}\right| d \tau \int_{t_{0}}^{s}\left\|\frac{\partial^{|L|}}{\partial x^{L}} \frac{\partial P}{\partial p_{0}}\right\|_{\infty} d \tau
\end{aligned}
$$

for $t_{0} \leqslant s<T$ and some constant $C>0$.

Remark. By definition, $Z_{i}(\tau)$ is a continuous process, so we can write $Z_{i}(\tau)$ instead of $Z_{i}(\tau-)$.

Proof. The first equation in the system (4.1) implies

$$
\left(\sup _{t_{0} \leqslant \tau \leqslant s}\left|\frac{\partial^{|J|} X(\tau)}{\partial p_{0}^{J}}\right|\right) \vee\left(\operatorname{Var}_{\left[t_{0}, s\right]} \frac{\partial^{|J|} X}{\partial p_{0}^{J}}\right) \leqslant \int_{t_{0}}^{s}\left|\frac{\partial^{|J|} P(\tau)}{\partial p_{0}^{J}}\right| d \tau
$$

for any $J \in \mathbb{N}_{0}^{d}$. 
By (4.3), $(\partial c / \partial x)=0$ for $|x|>K$. An application of Corollary 4.1 with $f=\left(\partial^{|\rho|} c_{i}^{(2)}(x) / \partial x^{\rho}\right)_{n, j}, \quad n, j=1, \ldots, d$, and condition (4.30) imply

$$
\sup _{t_{0} \leqslant \tau \leqslant s}\left\|\frac{\partial^{|\rho|} c_{i}^{(2)}(X(\tau))}{\partial x^{\rho}}\right\|_{\infty} \vee\left\|\operatorname{Var}_{\left[t_{0}, s\right]} \frac{\partial^{|\rho|} c_{i}^{(2)}(X)}{\partial x^{\rho}}\right\|_{\infty} \leqslant C_{1}
$$

for some constant $C_{1}>0$ and all $|\rho| \leqslant K$. Using the formula

$$
\sup _{t_{0} \leqslant \tau \leqslant s}\left|\prod_{l=1}^{r+2} a_{l}(\tau)\right| \vee \operatorname{Var}_{\left[t_{0}, s\right]}\left(\prod_{l=1}^{r+2} a_{l}\right) \leqslant(r+2) \prod_{l=1}^{r+2}\left(\sup _{t_{0} \leqslant \tau \leqslant s}\left|a_{l}(\tau)\right| \vee \operatorname{Var}_{\left[t_{0}, s\right]} a_{l}\right) \text {, }
$$

where $a_{l}: \mathbb{R} \rightarrow \mathbb{R}, l=1, \ldots, r+2$, we find

$$
\begin{aligned}
& \sup _{t_{0} \leqslant \tau \leqslant s}\left|Z_{i}(\tau)\right| \vee\left\|\operatorname{Var}_{\left[t_{0}, s\right]} Z_{i}\right\|_{\infty} \\
& \leqslant(r+2) C_{1} \int_{t_{0}}^{s}\left|\frac{\partial^{|\mu|} P(\tau)}{\partial p_{0}^{\mu}}\right| d \tau \ldots \int_{t_{0}}^{s}\left|\frac{\partial^{|\lambda|} P(\tau)}{\partial p_{0}^{\lambda}}\right| d \tau \int_{t_{0}}^{s}\left\|\frac{\partial^{|L|}}{\partial x^{L}} \frac{\partial P}{\partial p_{0}}\right\|_{\infty} d \tau .
\end{aligned}
$$

Since $X(\tau)$ is a continuous process, so is $Z(\tau)$ and (4.34) shows that $Z(\tau)$ is a $\mathrm{BV}$-process. Using integration by parts gives

$$
\int_{t_{0}}^{s} Z_{i}(\tau) d \eta_{i, \tau}=Z_{i}(s) \eta_{i, s}-Z_{i}\left(t_{0}\right) \eta_{i, t_{0}}-\int_{t_{0}}^{s} \eta_{i, \tau-} d Z_{i}(\tau)
$$

hence for $s<T$

$$
\begin{aligned}
& \left\|\int_{t_{0}}^{s} Z_{i}(\tau) d \eta_{i, \tau}\right\| \leqslant \\
& \quad \leqslant 2 \sup _{t_{0} \leqslant \tau \leqslant s}\left|\eta_{i, \tau}\right| \sup _{t_{0} \leqslant \tau \leqslant s}\left\|Z_{i}(\tau)\right\|_{\infty}+\sup _{t_{0} \leqslant \tau \leqslant s}\left|\eta_{i, \tau}\right|\left\|\operatorname{Var}_{\left[t_{0}, s\right]} Z_{i}\right\|_{\infty} .
\end{aligned}
$$

Recall that $\eta_{i, s}$ is a Lévy process. Therefore by Lemma 2.1 we have

$$
2 \sum_{i=0}^{d}\left(\sup _{t_{0} \leqslant \tau \leqslant s}\left|\eta_{i, s}\right|\right) \leqslant \theta_{t}+t \leqslant 2
$$

for $0 \leqslant t_{0} \leqslant s<T \leqslant \mathcal{R}$. Summing (4.35) over $i=0, \ldots, d$, and using (4.34) completes the proof.

\section{Proof of Lemma 4.2.}

Step 1. Let us choose and (in this step) fix a sequence $\left(j_{1}, j_{2}, \ldots\right) \in$ $\{1, \ldots, d\}^{\mathbb{N}}$. Write

$$
\begin{gathered}
\mathcal{A}_{0}(\tau)=\frac{\partial X(\tau)}{\partial p_{0}}, \mathcal{B}_{0}(\tau)=\frac{\partial P(\tau)}{\partial p_{0}}, \mathcal{A}_{m}(\tau)=\frac{\partial^{|J|} \mathcal{A}_{0}(\tau)}{\partial p_{0}^{J}}, \mathcal{B}_{m}(\tau)=\frac{\partial^{|J|} \mathcal{B}_{0}(\tau)}{\partial p_{0}^{J}}, \\
\mathcal{A}_{0}(\tau), \mathcal{B}_{0}(\tau), \mathcal{A}_{m}(\tau), \mathcal{B}_{m}(\tau) \in \mathbb{R}^{d \times d}, \text { where } \\
\quad J=\left(j_{1}, \ldots, j_{m}\right) \quad \text { and } \quad p_{0}=\left(p_{0,1}, \ldots, p_{0, d}\right) \in \mathbb{R}^{d}
\end{gathered}
$$


From equation (4.16) we get

$$
d_{\tau}\left(\begin{array}{c}
\mathcal{A}_{0}(\tau) \\
\mathcal{B}_{0}(\tau)
\end{array}\right)=\sum_{i=0}^{d} W_{i, \tau}\left(\begin{array}{c}
\mathcal{A}_{0}(\tau) \\
\mathcal{B}_{0}(\tau)
\end{array}\right) d \eta_{i, \tau}
$$

Differentiating (4.36) with respect to $p_{0, j_{1}}, \ldots, p_{0, j_{m}}$ we get the following system of SDE's

$$
\begin{aligned}
d_{\tau}\left(\begin{array}{c}
\mathcal{A}_{m}(\tau) \\
\mathcal{B}_{m}(\tau)
\end{array}\right) & =\sum_{i=0}^{d} W_{i, \tau}\left(\begin{array}{c}
\mathcal{A}_{m}(\tau) \\
\mathcal{B}_{m}(\tau)
\end{array}\right) d \eta_{i, \tau}-\sum_{i=0}^{d} \widetilde{A}_{m, i, \tau} d \eta_{i, \tau} \\
\mathcal{A}_{m}\left(t_{0}\right) & =\mathcal{B}_{m}\left(t_{0}\right)=0
\end{aligned}
$$

where $\widetilde{A}_{m, i, \tau} \in \mathbb{R}^{2 d \times d}$ is given by the following recurrence relation

$$
\widetilde{A}_{0, i, \tau}=0, \quad \widetilde{A}_{m, i, \tau}=-\frac{\partial W_{i, \tau}}{\partial p_{0, j_{m}}}\left(\begin{array}{c}
\mathcal{A}_{m-1}(\tau) \\
\mathcal{B}_{m-1}(\tau)
\end{array}\right)+\frac{\partial \widetilde{A}_{m-1, i, \tau}}{\partial p_{0, j_{m}}} \quad m>0 .
$$

The interchange of stochastic and ordinary differentials (with respect to the initial conditions) is possible since the coefficients of the system (4.36) are smooth enough cf. Protter [Pro, p. 245, Theorem 40]. Using (4.17) gives

$$
\widetilde{A}_{m, i, \tau}=\left(\begin{array}{c}
0 \\
A_{m, i, \tau}
\end{array}\right), \quad i=0, \ldots, d, \quad A_{m, i, \tau} \in \mathbb{R}^{d \times d}
$$

where

$$
A_{0, i, \tau}=0, \quad A_{m, i, \tau}=\frac{\partial c_{i}^{(2)}(X(\tau))}{\partial p_{0, j_{m}}} \mathcal{A}_{m-1}(\tau)+\frac{\partial A_{m-1, i, \tau}}{\partial p_{0, j_{m}}} \quad m>0 .
$$

Recall that $c_{i}^{(2)}(x)=\left(\partial^{2} c_{i}(x) / \partial x^{2}\right) \in \mathbb{R}^{d \times d}$. From (4.39) we find by induction

$$
A_{m, i, \tau}=\sum_{k=2}^{m+1} \frac{\partial^{m+1-k}}{\partial p_{0, j_{m}} \ldots \partial p_{0, j_{k}}}\left(\frac{\partial c_{i}^{(2)}(X(\tau))}{\partial p_{0, j_{k-1}}} \frac{\partial^{k-2} \mathcal{A}_{0}(\tau)}{\partial p_{0, j_{k-2}} \ldots \partial p_{0, j_{1}}}\right) .
$$

A solution of (4.37) is given by the following (formal) series expansion

$$
G=\sum_{k=1}^{\infty} G_{k}
$$

with

$$
G_{1}=-\sum_{i=0}^{d} \int_{t_{0}}^{t} \widetilde{A}_{m, i, \tau_{0}-} d \eta_{i, \tau_{0}}, \quad G_{k}=\sum_{i=0}^{d} \int_{t_{0}}^{t} W_{i, \tau} G_{k-1} d \eta_{i, \tau} \quad k \geqslant 2 .
$$

This can be seen as in the proof of Theorem 4.1 and (4.41) is a solution of (4.37) whenever it converges uniformly (on compact intervals) in $t$. 
Thus

$$
\left(\begin{array}{c}
\mathcal{A}_{m}(t) \\
\mathcal{B}_{m}(t)
\end{array}\right)=-\sum_{i=0}^{d} \int_{t_{0}}^{t} \widetilde{A}_{m, i, \tau_{0}-} d \eta_{i, \tau_{0}}-\sum_{k=1}^{\infty} \sum_{i_{1}, \ldots, i_{k}=0}^{d} \widetilde{I}_{i_{1}, \ldots, i_{k}, t}^{m}
$$

where

$$
\widetilde{I}_{i_{1}, \ldots, i_{k}, t}^{m}=\int_{t_{0}}^{t} W_{i_{k}, \tau_{k}} \ldots \int_{t_{0}}^{\tau_{2}-} W_{i_{1}, \tau_{1}}\left(\sum_{i=0}^{d} \int_{t_{0}}^{\tau_{1}-} \widetilde{A}_{m, i, \tau_{0}-} d \eta_{i, \tau_{0}}\right) d \eta_{i_{1}, \tau_{1}} \ldots d \eta_{i_{k}, \tau_{k}} .
$$

Since $W_{i_{1}, \tau} \widetilde{A}_{m, i, \tau}=0$ for $i_{1}=1, \ldots, d$, it follows that

$$
\widetilde{I}_{i_{1}, \ldots, i_{k}, t}^{m}=0 \quad \text { for } \quad i_{1}>0,
$$

i.e. the first integration in $\widetilde{I}_{i_{1}, \ldots, i_{k}, t}^{m}$ is trivial, which means $\ell_{1}>0$. Therefore

$$
\begin{aligned}
\left\|\sum_{k=1}^{\infty} \sum_{i_{1}, \ldots, i_{k}=0}^{d} \widetilde{I}_{i_{1}, \ldots, i_{k}, t}^{m}\right\|_{\infty} & =\left\|\sum_{k=1}^{\infty} \sum_{i_{2}, \ldots, i_{k}=0}^{d} \sum_{i_{1}=1}^{d} \widetilde{I}_{i_{1}, \ldots, i_{k}, t}^{m}\right\|_{\infty} \\
& \leqslant \sum_{k=1}^{\infty} 2^{k} \max \left\|\sum_{\left(i_{1}, \ldots, i_{k}\right) \in \mathcal{M}^{k}} \widetilde{I}_{i_{1}, \ldots, i_{k}, t}^{m}\right\|_{\infty},
\end{aligned}
$$

where $\mathcal{M}^{k}=\mathcal{M}_{1}^{k} \times \cdots \times \mathcal{M}_{k}^{k}, \mathcal{M}_{j}^{k}=\{1, \ldots, d\}$ or $\mathcal{M}_{j}^{k}=\{0\}$ for $j>1$ and the maximum is taken over all $\mathcal{M}^{k}$ such that $\mathcal{M}_{1}^{k}=\{0\}$. Also observe that formula (3.10) still holds if $I_{0, t}$ is not a square $M \times M$ matrix, but rectangular of the form $I_{0, t} \in \mathbb{R}^{M \times N}$ and any $N \in \mathbb{N}$. An application of Proposition 3.1 to the matrices $K^{-1} W_{j, \tau} \in \mathbb{R}^{2 d \times 2 d}, \quad j=1, \ldots, d$, with $I_{0, t}=\sum_{i=0}^{d} \int_{t_{0}}^{t} \widetilde{A}_{m, i, \tau_{0}-} d \eta_{i, \tau_{0}} \in \mathbb{R}^{2 d \times d}$ shows

$$
\left\|\sum_{\left(i_{1}, \ldots, i_{k}\right) \in \mathcal{M}^{k}} \widetilde{I}_{i_{1}, \ldots, i_{k}, t}^{m}\right\|_{\infty} \leqslant K^{k} b_{k} D_{t}^{\mathfrak{m}}\left\{2 d\left(t-t_{0}\right)\right\}^{k-\mathfrak{m}}\left\|I_{0, t}^{*}\right\|_{\infty}
$$

where the $b_{k}$ 's are given by (3.11). Thus for $t<T$

$$
\begin{aligned}
\left\|\mathcal{A}_{m}(t)\right\|_{\infty} \vee & \left\|\mathcal{B}_{m}(t)\right\|_{\infty} \\
\leqslant & \sum_{i=0}^{d}\left\|\int_{t_{0}}^{t} \widetilde{A}_{m, i, \tau_{0}-} d \eta_{i, \tau_{0}}\right\|_{\infty} \\
& +\sum_{k=1}^{\infty} 2^{k} K^{k} b_{k} D_{t}^{\mathfrak{m}}\left\{2 d\left(t-t_{0}\right)\right\}^{k-\mathfrak{m}} \sup _{t_{0} \leqslant s \leqslant t} \sum_{i=0}^{d}\left\|\int_{t_{0}}^{s} \widetilde{A}_{m, i, \tau_{0}-} d \eta_{i, \tau_{0}}\right\|_{\infty} \\
\leqslant & \sup _{t_{0} \leqslant s \leqslant t} \sum_{i=0}^{d}\left\|\int_{t_{0}}^{s} \widetilde{A}_{m, i, \tau_{0}-} d \eta_{i, \tau_{0}}\right\|_{\infty} \widehat{\Phi}\left(D_{t}+2 d\left(t-t_{0}\right)\right),
\end{aligned}
$$

where $\widehat{\Phi}(x)=1+\sum_{k=1}^{\infty} 2^{k} K^{k} b_{k} x^{k}, D_{\tau}$ and $b_{k}$ are given by (3.8) and (3.11) respectively. 
By $(4.23), \quad \widehat{\Phi}\left(D_{t}+2 d\left(t-t_{0}\right)\right) \leqslant \widehat{\Phi}\left(2 d^{2}\left(\widetilde{K}_{2}+4\right)+2 d\right)=O(1)$ for $t<T$, so we get from (4.38) arrive at

$$
\left\|\frac{\partial^{|J|} \mathcal{B}_{0}(t)}{\partial p_{0}^{J}}\right\|_{\infty}=\left\|\mathcal{B}_{m}(t)\right\|_{\infty}=O(1) \sup _{t_{0} \leqslant s \leqslant t} \sum_{i=0}^{d}\left\|\int_{t_{0}}^{s} A_{m, i, \tau_{0}-} d \eta_{i, \tau_{0}}\right\|_{\infty} .
$$

Step 2. Now we apply induction in $m$ to prove

$$
\mathcal{A}_{m}=O\left(\left(t-t_{0}\right)^{m+2}\right), \quad \mathcal{B}_{m}=O\left(\left(t-t_{0}\right)^{m+1}\right)
$$

for any $J \in \mathbb{N}_{0}^{m}$, which is equivalent the assertion of the lemma.

Let us first check the claim for $m=1$. From (4.40) we find

$$
A_{1, i, \tau}=\sum_{j=1}^{d} \frac{\partial c_{i}^{(2)}(X(\tau))}{\partial x_{j}} \frac{\partial X_{j}(\tau)}{\partial p_{0, j_{1}}} \frac{\partial X(\tau)}{\partial p_{0}}
$$

An application of Lemma 4.3 with $r=1, i_{1}=j, \rho=e_{j}, \mu=e_{j_{1}}$ (see definition (3.36)) and $L=0$ shows

$$
\left\|\int_{t_{0}}^{s} \frac{\partial c_{i}^{(2)}(X(\tau))}{\partial x_{j}} \frac{\partial X_{j}(\tau)}{\partial p_{0, j_{1}}} \frac{\partial X(\tau)}{\partial p_{0}} d \eta_{i, \tau}\right\|_{\infty}=O(1)\left(\int_{t_{0}}^{s}\left\|\frac{\partial P(\tau)}{\partial p_{0}}\right\|_{\infty} d \tau\right)^{2} .
$$

By (4.5) we know $\left\|\partial P / \partial p_{0}\right\|_{\infty}=O(1)$. Hence

$$
\sup _{t_{0} \leqslant s \leqslant t} \sum_{i=0}^{d}\left\|\int_{t_{0}}^{s} A_{1, i, \tau-} d \eta_{i, \tau}\right\|_{\infty}=O\left(\left(t-t_{0}\right)^{2}\right) .
$$

Combining this and (4.42) gives $\mathcal{B}_{1}=O\left(\left(t-t_{0}\right)^{2}\right)$. Using (4.1) we obtain $\mathcal{A}_{1}=O\left(\left(t-t_{0}\right)^{3}\right)$.

We now assume that (4.43) holds for $1, \ldots, m-1$. From (4.40) we find that $A_{m, i, \tau}$ is a linear combination of the $Z_{i}(\tau)=Z_{i, i_{1}, \ldots, i_{r}, \rho, \mu, \ldots, \lambda, L}(\tau)$ given by (4.33) with

$$
|\rho|=r \leqslant m, \quad|\mu|+\ldots+|\lambda|+|L|=m .
$$

By the induction assumption and by (4.5) we have $\left\|\partial^{|L|} \mathcal{B}_{0}(\tau) / \partial x^{L}\right\|_{\infty}=$ $O\left(\left(t-t_{0}\right)^{|L|}\right)$ and

$$
\left|\frac{\partial^{|\mu|} P(\tau)}{\partial p_{0}^{\mu}}\right|=O\left(\left(t-t_{0}\right)^{\phi(|\mu|)}\right), \quad \ldots, \quad\left|\frac{\partial^{|\lambda|} P(\tau)}{\partial p_{0}^{\lambda}}\right|=O\left(\left(t-t_{0}\right)^{\phi(|\lambda|)}\right),
$$

where $\phi: \mathbb{N} \rightarrow \mathbb{N}$ such that $\phi(1)=0, \phi(n)=n$ for all $n>1$. (Note that the choice of $\phi$ allows us to combine the formulae (4.5) and (4.32), which show 
different behaviour for $|\mu|=1$ resp. $|\mu|>1$.) An application of Lemma 4.3 shows

$$
\sum_{i=0}^{d}\left\|\int_{t_{0}}^{s} A_{m, i, \tau-} d \eta_{i, \tau}\right\|_{\infty}=O\left(\left(t-t_{0}\right)^{\gamma}\right)
$$

where

$$
\gamma=\phi(|\mu|)+1+\ldots+\phi(|\lambda|)+1+|L|+1
$$

Since $1+\phi(n) \geqslant n$ we conclude from (4.44) that

$$
\gamma \geqslant|\mu|+\ldots+|\lambda|+|L|+1=m+1
$$

Combining this, (4.45) and (4.42) we have $\mathcal{B}_{m}=O\left(\left(t-t_{0}\right)^{m+1}\right)$. The assertion for $\mathcal{A}_{m}$ follows again from the first equation in (4.1).

\section{The method of stochastic characteristics}

As before we denote by $(X, P)=\left(X\left(t, t_{0}, x_{0}, p_{0}\right), P\left(t, t_{0}, x_{0}, p_{0}\right)\right)$ the solution of the Hamilton system

$$
\left\{\begin{array}{l}
d x=\frac{\partial H}{\partial p} d t \\
d p=-\frac{\partial H}{\partial x} d t-\frac{\partial c}{\partial x} d \xi_{t}
\end{array}\right.
$$

with initial condition $\left(x_{0}, p_{0}\right) \in \mathbb{R}^{2 d}$ at $t=t_{0}$, where

$$
H: \mathbb{R}^{d} \times \mathbb{R}^{d} \rightarrow \mathbb{R}, \quad c: \mathbb{R}^{d} \rightarrow \mathbb{R}^{d} .
$$

We shall say that $H$ and $c$ satisfy property (D1) if

There exists a stopping time $T>0$ a.s. such that

(D1) for any $0 \leqslant t_{0}<t<T, \forall x_{0} \in \mathbb{R}^{d}$ the map

$\mathfrak{D}_{1}: \mathbb{R}^{d} \rightarrow \mathbb{R}^{d}, \quad p_{0} \mapsto X\left(t, t_{0}, x_{0}, p_{0}\right)$

is a diffeomorphism.

Next we shall say that $H, c$ and $S_{0}: \mathbb{R}^{d} \rightarrow \mathbb{R}$ satisfy property (D2) if

There exists a stopping time $T>0$ a.s. such that

for any $0 \leqslant t_{0}<t<T$ the map

$\mathfrak{D}_{2}: \mathbb{R}^{d} \rightarrow \mathbb{R}^{d}, \quad x_{0} \mapsto X\left(t, t_{0}, x_{0}, \nabla S_{0}\left(x_{0}\right)\right)$

is a diffeomorphism.

Remark. In the literature on Burgers turbulence, the map $\mathfrak{D}_{2}$ is called Lagrangian function, and its inverse $\mathfrak{D}_{2}^{-1}$ is called the inverse Lagrangian function [Ber2]. 
In the following statement we summarise the main results of the previous section.

Theorem 5.1 Let $S_{0}: \mathbb{R}^{d} \rightarrow \mathbb{R}$ be a twice differentiable function such that

$$
\frac{\partial^{2} S_{0}(x)}{\partial x^{2}} \geqslant \Lambda \quad \text { for some symmetric } \Lambda \in \mathbb{R}^{d \times d}, \forall x \in \mathbb{R}^{d},
$$

$H(x, p)=(1 / 2) p^{2}-V(x)$ and the conditions of the Theorem 4.1 hold. Then (D1) and (D2) are satisfied.

Proof. Theorem 4.1 immediately implies (D1).

Using formulae (4.5), (4.6) we deduce from

$$
\begin{aligned}
& \frac{\partial X\left(t, t_{0}, x_{0}, \nabla S_{0}\left(x_{0}\right)\right)}{\partial x_{0}} \\
& \quad=\left.\frac{\partial X\left(t, t_{0}, x_{0}, p_{0}\right)}{\partial x_{0}}\right|_{p_{0}=\nabla S_{0}\left(x_{0}\right)}+\frac{\partial X\left(t, t_{0}, x_{0}, \nabla S_{0}\left(x_{0}\right)\right)}{\partial p_{0}} \frac{\partial^{2} S_{0}\left(x_{0}\right)}{\partial x_{0}^{2}}
\end{aligned}
$$

that

$$
\frac{\partial X\left(t, t_{0}, x_{0}, \nabla S_{0}\left(x_{0}\right)\right)}{\partial x_{0}}=E_{d}+O\left(t-t_{0}\right)+\left[\left(t-t_{0}\right)+O\left(t-t_{0}\right)\right] \frac{\partial^{2} S_{0}\left(x_{0}\right)}{\partial x_{0}^{2}}
$$

and so there exist a constant $C_{1}>0$ such that

$$
\frac{\partial X\left(t, t_{0}, x_{0}, \nabla S_{0}\left(x_{0}\right)\right)}{\partial x_{0}} \geqslant \frac{1}{2} E_{d} \quad \text { for } \quad 0 \leqslant t_{0} \leqslant t<T \wedge C_{1}
$$

Therefore the map $\mathfrak{D}_{2}: x_{0} \mapsto X\left(t, t_{0}, x_{0}, \nabla S_{0}\left(x_{0}\right)\right)$ is a local diffeomorphism. Along the same lines as in the proof of Theorem 4.1 we conclude that $\mathfrak{D}_{2}$ is a global diffeomorphism.

Recall that the notations $X(\tau), P(\tau)$ were introduced in (4.7). To each pair $(X(\tau), P(\tau))$ of solutions of (5.1) there corresponds the action function defined by the formula

$$
\sigma\left(t, t_{0}, x_{0}, p_{0}\right)=\int_{t_{0}}^{t}\left[P(\tau) \frac{\partial X(\tau)}{\partial \tau}-H(X(\tau), P(\tau))\right] d \tau-\int_{t_{0}}^{t} c(X(\tau)) d \xi_{\tau}
$$

If (D1) holds, the inverse map $p_{0}=p_{0}\left(t, t_{0}, x, x_{0}\right)$ to $\mathfrak{D}_{1}$ exists, i.e.

$$
X\left(t, t_{0}, x_{0}, p_{0}\left(t, t_{0}, x, x_{0}\right)\right)=x, \quad t>t_{0}, \quad x \in \mathbb{R}^{n},
$$

and we can define locally (for $0 \leqslant t_{0}<t<T$ ) the two-point function

$$
S\left(t, t_{0}, x, x_{0}\right)=\sigma\left(t, t_{0}, x_{0}, p_{0}\left(t, t_{0}, x, x_{0}\right)\right) .
$$


Notation. We set

$$
p\left(t, t_{0}, x, x_{0}\right)=P\left(t, t_{0}, x_{0}, p_{0}\left(t, t_{0}, x, x_{0}\right)\right)
$$

and

$$
x(\tau)=X\left(\tau, t_{0}, x_{0}, p_{0}\left(t, t_{0}, x, x_{0}\right)\right), \quad p(\tau)=P\left(\tau, t_{0}, x_{0}, p_{0}\left(t, t_{0}, x, x_{0}\right)\right) .
$$

Finally, we put $X\left(\tau, x_{0}, p_{0}\right)=X\left(\tau, 0, x_{0}, p_{0}\right), P\left(\tau, x_{0}, p_{0}\right)=P\left(\tau, 0, x_{0}, p_{0}\right)$, $p_{0}\left(\tau, 0, x_{0}, p_{0}\right)=p_{0}\left(\tau, x_{0}, p_{0}\right), \sigma\left(t, x_{0}, p_{0}\right)=\sigma\left(t, 0, x_{0}, p_{0}\right)$ and $S\left(t, x, x_{0}\right)=$ $S\left(t, 0, x, x_{0}\right)$. We will use this notation throughout the rest of this paper.

The following results (and their proofs) are stochastic versions of the well known method of characteristics for solving the Hamilton-Jacobi equation (see e.g. [K3]).

Theorem 5.2 Let $H(x, p)$ and $c(x)$ satisfy (D1). The function $(t, x) \mapsto$ $S\left(t, t_{0}, x, x_{0}\right)$, as a function of the variables $(t, x)$, satisfies the HamiltonJacobi equation

$$
d S+H\left(x, \frac{\partial S}{\partial x}\right) d t+c(x) d \xi_{t}=0
$$

in the domain $\left(t_{0}, T\right) \times \mathbb{R}^{d}$ for the stopping time $T$ with $\mathbb{P}(T>0)=1$. Moreover, we have

$$
\frac{\partial S}{\partial x}\left(t, t_{0}, x, x_{0}\right)=p\left(t, t_{0}, x, x_{0}\right), \quad \frac{\partial S}{\partial x_{0}}\left(t, t_{0}, x, x_{0}\right)=-p_{0}\left(t, t_{0}, x, x_{0}\right) .
$$

Proof. Without loss of generality we may assume that $t_{0}=0$.

Step 1. We start with the proof of the first relation in (5.9). This equality can be rewritten as

$$
\frac{\partial S}{\partial x}\left(t, X\left(t, x_{0}, p_{0}\right), x_{0}\right)=P\left(t, x_{0}, p_{0}\right)
$$

which is, by (5.5),

$$
\frac{\partial \sigma}{\partial p_{0}}\left(t, x_{0}, p_{0}\right) \frac{\partial p_{0}}{\partial x}\left(t, X\left(t, x_{0}, p_{0}\right), x_{0}\right)=P\left(t, x_{0}, p_{0}\right)
$$

Due to (5.4),

$$
\left(\frac{\partial p_{0}}{\partial x}\left(t, X\left(t, x_{0}, p_{0}\right), x_{0}\right)\right)^{-1}=\frac{\partial X}{\partial p_{0}}\left(t, x_{0}, p_{0}\right) .
$$

It follows that the first equation in (5.9), using (5.10), has the form

$$
\frac{\partial \sigma}{\partial p_{0}}\left(t, x_{0}, p_{0}\right)=P\left(t, x_{0}, p_{0}\right) \frac{\partial X}{\partial p_{0}}\left(t, x_{0}, p_{0}\right) .
$$


Since $X(t), \partial X(t) / \partial p_{0}$ are continuous and of bounded variation, we get from Itô's formula that

$$
d P \frac{\partial X}{\partial p_{0}}=P d \frac{\partial X}{\partial p_{0}}+\frac{\partial X}{\partial p_{0}} d P .
$$

The left-hand side of (5.12) can be expressed using (5.3). Together with (5.13) we calculate that its Itô differential gives

$$
\frac{\partial}{\partial p_{0}}\left(P \frac{\partial X}{\partial t}-H\right) d t-\frac{\partial c(X)}{\partial p_{0}} d \xi_{t}=P d \frac{\partial X}{\partial p_{0}}+\frac{\partial X}{\partial p_{0}} d P
$$

Notice that we need the fact that

$$
\frac{\partial}{\partial p_{0}} \int_{0}^{t} \frac{\partial c(X(\tau))}{\partial x} d \xi_{\tau}=\sum_{i=1}^{d} \int_{0}^{t} \frac{\partial^{2} c_{i}(X(\tau))}{\partial p_{0} \partial x} d \xi_{i, \tau}
$$

which is justified by a special case of Theorem $36.9[\mathrm{M}]$, p. 258. Since by $(4.1) d P=(\partial H / \partial x) d t+(\partial c / \partial x) d \xi_{t}$, we find

$$
\begin{aligned}
& \frac{\partial P}{\partial p_{0}} \frac{\partial X}{\partial t} d t+P \frac{\partial^{2} X}{\partial p_{0} \partial t} d t-\frac{\partial H}{\partial x} \frac{\partial X}{\partial p_{0}} d t-\frac{\partial H}{\partial p} \frac{\partial P}{\partial p_{0}} d t-\frac{\partial X}{\partial p_{0}} \frac{\partial c}{\partial x} d \xi_{t} \\
& \quad=P \frac{\partial^{2} X}{\partial p_{0} \partial t} d t-\frac{\partial X}{\partial p_{0}}\left(\frac{\partial H}{\partial x} d t+\frac{\partial c}{\partial x} d \xi_{t}\right) .
\end{aligned}
$$

As $\partial X / \partial t=P$ we find that (5.14) holds for all $t<T(\omega)$, and the first part of (5.9) is established.

Step 2. Using (5.5) we get

$$
\frac{\partial S}{\partial x_{0}}=\frac{\partial \sigma}{\partial x_{0}}+\frac{\partial \sigma}{\partial p_{0}} \frac{\partial p_{0}\left(t, x, x_{0}\right)}{\partial x_{0}}
$$

and so, by (5.4), we rewrite the second formula in (5.9) as

$$
\frac{\partial \sigma}{\partial x_{0}}-\frac{\partial \sigma}{\partial p_{0}}\left(\frac{\partial X}{\partial p_{0}}\right)^{-1} \frac{\partial X}{\partial x_{0}}=-p_{0}
$$

The relation

$$
\frac{\partial}{\partial \alpha}\left(\left[P(\tau) \frac{\partial X(\tau)}{\partial \tau}-H(X(\tau), P(\tau))\right] d \tau-c(X(\tau)) d \xi_{\tau}\right)=d_{\tau}\left(P(\tau) \frac{\partial X(\tau)}{\partial \alpha}\right)
$$

where $\alpha=x_{0}$ or $\alpha=p_{0}$, and definition (5.3) imply

$$
\frac{\partial \sigma}{\partial x_{0}}=\left.\left(P(\tau) \frac{\partial X(\tau)}{\partial x_{0}}\right)\right|_{0} ^{t}, \quad \frac{\partial \sigma}{\partial p_{0}}=\left.\left(P(\tau) \frac{\partial X(\tau)}{\partial p_{0}}\right)\right|_{0} ^{t} .
$$


Using (5.16) and the fact that

$$
\left.\frac{\partial X(\tau)}{\partial x_{0}}\right|_{\tau=0}=1,\left.\quad \frac{\partial X(\tau)}{\partial p_{0}}\right|_{\tau=0}=0
$$

gives (5.15).

Step 3. To prove (5.8), let us first rewrite it as

$$
d \sigma\left(t, x_{0}, p_{0}\right)+\frac{\partial \sigma}{\partial p_{0}} d p_{0}+H\left(x, p\left(t, x_{0}, x\right)\right) d t+c(x) d \xi_{t}=0
$$

Because of (5.3) we find

$$
\begin{aligned}
& P\left(t, x_{0}, p_{0}\right) \frac{\partial X}{\partial t}\left(t, x_{0}, p_{0}\right) d t-H\left(X\left(t, x_{0}, p_{0}\right), P\left(t, x_{0}, p_{0}\right)\right) d t \\
& \quad-\quad c\left(X\left(t, x_{0}, p_{0}\right)\right) d \xi_{t}+\frac{\partial \sigma}{\partial p_{0}} d p_{0}+H\left(x, p\left(t, x, x_{0}\right)\right) d t+c(x) d \xi_{t}=0
\end{aligned}
$$

By construction, $X\left(\tau, x_{0}, p_{0}\right)=x, P\left(t, x_{0}, p_{0}\right)=p$ and expressing $\partial \sigma / \partial p_{0}$ by (5.12) gives

$$
P\left(t, x_{0}, p_{0}\right) \frac{\partial X}{\partial t}\left(t, x_{0}, p_{0}\right) d t+P\left(t, x_{0}, p_{0}\right) \frac{\partial X}{\partial p_{0}}\left(t, x_{0}, p_{0}\right) d p_{0}=0 .
$$

Differentiating (5.4) with respect to $t$ we get

$$
d X\left(t, x_{0}, p_{0}\left(t, x_{0}, x\right)\right)=\frac{\partial X}{\partial t}\left(t, x_{0}, p_{0}\right) d t+\frac{\partial X}{\partial p_{0}}\left(t, x_{0}, p_{0}\right) d p_{0}=0
$$

Thus (5.17) is always satisfied and (5.8) follows.

Corollary 5.1 Under the assumption of the Theorem 5.2 we have for $0 \leqslant$ $t_{0}<t<T$

$$
\begin{aligned}
\frac{\partial^{2} S\left(t, t_{0}, x, x_{0}\right)}{\partial x^{2}} & =\frac{1}{t-t_{0}}\left(E_{d}+O\left(t-t_{0}\right)\right), \\
\frac{\partial^{2} S\left(t, t_{0}, x, x_{0}\right)}{\partial x_{0}^{2}} & =\frac{1}{t-t_{0}}\left(E_{d}+O\left(t-t_{0}\right)\right), \\
\frac{\partial^{2} S\left(t, t_{0}, x, x_{0}\right)}{\partial x \partial x_{0}} & =-\frac{1}{t-t_{0}}\left(E_{d}+O\left(t-t_{0}\right)\right),
\end{aligned}
$$

where $O(\cdot)$ is uniform with respect to $x_{0}, x$. 
Proof. Assume again that $t_{0}=0$. From (5.9) and (5.11) we deduce the equality

$$
\frac{\partial^{2} S\left(t, x, x_{0}\right)}{\partial x^{2}}=\frac{\partial P}{\partial p_{0}}\left(t, x_{0}, p_{0}\right)\left(\frac{\partial X}{\partial p_{0}}\left(t, x_{0}, p_{0}\right)\right)^{-1} .
$$

Now (4.5), (4.6) imply the first formula in Corollary 5.1. The same argument can be used to prove the remaining formulae.

Theorem 5.3 We assume that $H(x, p), c(x)$ and $S_{0}(x)$ satisfy conditions (D1), (D2). Then for $0 \leqslant t_{0}<t<T(\omega)$ the formula

$$
S\left(t, t_{0}, x\right)=S_{0}\left(x_{0}\right)+\int_{t_{0}}^{t}\left(\widetilde{p}(\tau) d \widetilde{x}(\tau)-H(\widetilde{x}(\tau), \widetilde{p}(\tau)) d \tau-c(\widetilde{x}(\tau)) d \xi_{\tau}\right)
$$

(where the integral is taken along the trajectory $\widetilde{x}(\tau)=X\left(\tau, t_{0}, x_{0}, \nabla S_{0}\left(x_{0}\right)\right.$ ), $\widetilde{p}(\tau)=P\left(\tau, t_{0}, x_{0}, \nabla S_{0}\left(x_{0}\right)\right)$ and $x_{0}=x_{0}\left(t, t_{0}, x\right)$ is the inverse map of $\left.\mathfrak{D}_{2}\right)$ gives the unique classical solution of the Cauchy problem for the equation

$$
d S+H\left(x, \frac{\partial S}{\partial x}\right) d t+c(x) d \xi_{t}=0
$$

with initial function $S_{0}(x)$. One can rewrite formula (5.21) in the equivalent form

$$
S\left(t, t_{0}, x\right)=\left.\left(S_{0}\left(x_{0}\right)+S\left(t, t_{0}, x, x_{0}\right)\right)\right|_{x_{0}=x_{0}\left(t, t_{0}, x\right)} .
$$

Proof. The definition of the two-point function (5.5) implies the equivalence of (5.21) and (5.23). From the system (5.1) we see that $X\left(t, t_{0}, x_{0}, p_{0}\right)$ is continuous in $t$ and, using the implicit function theorem, we obtain from (D2) that $x_{0}=x_{0}\left(t, t_{0}, x\right)$ is continuous in $t$. So, $\left[x_{0}, x_{0}\right]=0$ and Itô's formula for this equation gives

$$
\begin{aligned}
& d_{t} S\left(t, t_{0}, x\right) \\
& \quad=\nabla S_{0}\left(x_{0}\right) d_{t} x_{0}\left(t, t_{0}, x\right)+\frac{\partial S\left(t, t_{0}, x, x_{0}\right)}{\partial t} d t+\frac{\partial S\left(t, t_{0}, x, x_{0}\right)}{\partial x_{0}} d_{t} x_{0}\left(t, t_{0}, x\right) \\
& \quad=\frac{\partial S\left(t, t_{0}, x, x_{0}\right)}{\partial t} d t .
\end{aligned}
$$

In the last equality we used $\nabla S_{0}\left(x_{0}\right)=p_{0}$ in conjunction with (5.9). From Theorem 5.2 we know that

$$
\frac{\partial S\left(t, t_{0}, x, x_{0}\right)}{\partial t} d t=d_{t} S\left(t, t_{0}, x, x_{0}\right)=-H\left(x, \frac{\partial S\left(t, t_{0}, x, x_{0}\right)}{\partial x}\right) d t-c(x) d \xi_{t},
$$

and the theorem follows. 


\section{Appendix}

In the proof of Proposition 3.1 we used some technical estimates which are not directly related to the arguments of Section 3 .

Lemma A.1 For $y_{1}, \ldots, y_{n} \geqslant 1$ one has

$$
\prod_{l=1}^{n}\left(y_{1}+\cdots+y_{l}\right) \Gamma\left(y_{l}\right)>\frac{1}{2^{n}} \Gamma(n+1)\left(\Gamma\left(\frac{y_{1}+\cdots+y_{n}}{n}+1\right)\right)^{n} .
$$

Proof. Since $y_{1}+\cdots+y_{l}>l-1$ we easily see

$$
\prod_{l=1}^{n}\left(y_{1}+\cdots+y_{l}\right)>\frac{n !}{2^{n}} \prod_{l=1}^{n} \frac{y_{1}+\cdots+y_{l}+l-1}{l} .
$$

We are going to prove by induction that

$$
\prod_{l=1}^{n} \frac{y_{1}+\cdots+y_{l}+l-1}{l} \geqslant \prod_{\substack{1 \leqslant l \leqslant n \\ i_{l}>0}} \prod_{s=1}^{i_{l}}\left(y_{l}+s-1\right)
$$

for some $i_{1}, \ldots, i_{n} \geqslant 0$ such that $i_{1}+\cdots+i_{n}=n$. Indeed, for $n=1$ we take $i_{1}=1$. Assume that (A.2) is true for $n-1$ and let $B=\min \left\{y_{1}+\right.$ $\left.i_{1}, \ldots, y_{n-1}+i_{n-1}, y_{n}\right\}$. Then

$$
\frac{y_{1}+\cdots+y_{n}+n-1}{n}=\frac{\left(y_{1}+i_{1}\right)+\cdots+\left(y_{n-1}+i_{n-1}\right)+y_{n}}{n} \geqslant B .
$$

If $B=y_{r}+i_{r}$ for some $1 \leqslant r \leqslant n-1$ we take $j_{r}=i_{r}+1, j_{s}=i_{s}$ for $s \neq r$ and $j_{n}=0$. Otherwise we put $j_{n}=1, j_{s}=i_{s}, s=1, \ldots, n-1$. Then the inequality (A.2) holds for $\left(j_{1}, \ldots, j_{n}\right)$, and all $n$.

Combining (A.1) and (A.2) we get

$$
\prod_{l=1}^{n}\left(y_{1}+\cdots+y_{l}\right) \Gamma\left(y_{l}\right)>\frac{n !}{2^{n}} \prod_{l=1}^{n} \Gamma\left(y_{l}+i_{l}\right) .
$$

The Lemma follows since the Gamma-function is log-convex, cf. [A].

Lemma A.2 We define $q_{k}$ by the following recursion formula

$$
q_{0}=1, \quad q_{k}=\sum_{m=0}^{k-1} \frac{3}{(k-m) m !} q_{k-m-1}, \quad k \in \mathbb{N} .
$$

Then

$$
q_{k} \leqslant \frac{\left(2^{6}\right)^{k}}{(\ln (k+1))^{\frac{k}{2}}}, \quad k \in \mathbb{N}
$$


Proof. With $a_{k, l}=3 /[(l+1)(k-l-1)$ !] formula (A.3) reads

$$
q_{k}=\sum_{l=0}^{k-1} a_{k, l} q_{l}, \quad k \geqslant 1
$$

Clearly,

$$
q_{k}=\sum_{\substack{i_{1}, \ldots, i_{n}, k=i_{0}>\ldots>i_{n}=0}} a_{i_{0}, i_{1}} \ldots a_{i_{n-1}, i_{n}}
$$

Notice that

$$
a_{i_{0}, i_{1}} \ldots a_{i_{n-1}, i_{n}}=\prod_{s=0}^{n-1} \frac{3}{\left(i_{s+1}+1\right) \Gamma\left(i_{s}-i_{s+1}\right)} .
$$

Lemma $A .1$ implies that for $b_{1}, \ldots, b_{r} \geqslant 1$

$$
\begin{gathered}
\Gamma\left(b_{n}\right) \prod_{l=1}^{n-1}\left(b_{1}+\cdots+b_{l}\right) \Gamma\left(b_{l}\right) \geqslant \frac{1}{2^{n}} \frac{\Gamma(n+1)}{b_{1}+\cdots+b_{n}}\left(\Gamma\left(\frac{b_{1}+\cdots+b_{n}}{n}+1\right)\right)^{n} . \\
\text { If } b_{l}=i_{n-l}-i_{n-l+1}, l=2, \ldots, n, b_{1}=i_{n-1}-i_{n}+1, \text { then } \\
b_{1}+\cdots+b_{n}=i_{0}-i_{n}+1=k+1,
\end{gathered}
$$

and it follows that

$$
B:=\Gamma\left(i_{0}-i_{1}\right) \prod_{s=1}^{n-1}\left(i_{s}+1\right) \Gamma\left(i_{s}-i_{s+1}\right) \geqslant \frac{1}{2^{n}} \frac{\Gamma(n+1)}{k+1}\left(\Gamma\left(\frac{k+1}{n}+1\right)\right)^{n} .
$$

Recall that $n \leqslant k-1$. Because of the inequalities $\Gamma(b+1) \geqslant b^{\frac{b}{2}}$ for $b \geqslant 2$, $\Gamma(b+1) \geqslant 2^{-1} b^{\frac{b}{2}}$ for $1 \leqslant b \leqslant 2$ and $n^{n-k} k^{k} \geqslant 2^{-k}(\ln (k+1))^{k}$ we have

$$
B \geqslant \frac{1}{4^{n}} \frac{n^{\frac{n}{2}}}{k+1}\left(\frac{k}{n}\right)^{\frac{k}{2}} \geqslant \frac{1}{k 4^{k}}\left(n^{n-k} k^{k}\right)^{\frac{1}{2}} \geqslant \frac{1}{k 8^{k}}(\ln (k+1))^{\frac{k}{2}} .
$$

Consequently, the right-hand side of (A.6) does not exceed $3^{n} B^{-1}$ and so

$$
q_{k} \leqslant 2^{k} \max _{k=i_{0}>\ldots>i_{n}=0} a_{i_{0}, i_{1}} \ldots a_{i_{n-1}, i_{n}} \leqslant \frac{k(48)^{k}}{(\ln (k+1))^{\frac{k}{2}}},
$$

where we used that the number of terms in (A.5) is equal to

$$
\sum_{n=1}^{k}\left(\begin{array}{l}
k-1 \\
n-1
\end{array}\right)=2^{k-1}
$$

Lemma A.3 For any $m, k, \alpha_{1}, \ldots, \alpha_{n} \in \mathbb{N}$ with

$$
\alpha_{1}+\cdots+\alpha_{n}+m=k, \quad m \geqslant n-1
$$

we have

(A.8) $\quad\left\{\ln \left(\alpha_{1}+1\right)\right\}^{\frac{\alpha_{1}}{2}} \ldots\left\{\ln \left(\alpha_{n}+1\right)\right\}^{\frac{\alpha_{n}}{2}} m ! \geqslant 2^{-k}\{\ln (\ln (k+2))\}^{\frac{k}{16}}$. 
Proof. Denote the left-hand side of (A.8) by $\mathbf{I}$ and observe that

$$
\mathbf{I} \geqslant(\sqrt{\ln 2})^{n} \geqslant 2^{-k}
$$

Since

$$
\{\ln (\ln (k+2))\}^{\frac{k}{8}} \leqslant 1 \quad \text { for } k \leqslant 10,
$$

(A.8) holds for $k \leqslant 10$. If $k>10$ we get from (A.7)

$$
\frac{\alpha_{1}+\cdots+\alpha_{n}}{n} \geqslant \frac{k-m}{m+1}
$$

and so, using the log-convexity of $f(x)=\{\ln (x+1)\}^{\frac{x}{2}}$,

$$
\mathbf{I} \geqslant\left\{\ln \left(\frac{\alpha_{1}+\cdots+\alpha_{n}}{n}+1\right)\right\}^{\frac{\alpha_{1}+\cdots+\alpha_{n}}{2}} m ! \geqslant\left\{\ln \left(\frac{k-m}{m+1}+1\right)\right\}^{\frac{k-m}{2}} m !
$$

Applying Lemma A.4, completes the proof.

Lemma A.4 For $1 \leqslant m<k, k \geqslant 10, m, k \in \mathbb{N}$ one has

$$
\left\{\ln \left(\frac{k+1}{m+1}\right)\right\}^{\frac{k-m}{2}} \Gamma(m+1) \geqslant 2^{-k}\{\ln (\ln (k+2))\}^{\frac{k}{16}} .
$$

Proof. We write $\mathbf{I}$ for the left-hand side of (A.9) and set $x_{0}=(\ln k)^{-1} k$.

Case 1. $x_{0}<m<k$. We split the proof into three steps. Take $x_{0}<x<k$, $x \in \mathbb{R}$.

Step 1. Since $(k+1) /(x+1)<k / x<\ln k$ we get

$$
\ln \left(\frac{k+1}{x+1}\right)<\ln \left(\frac{k}{x}\right)<\ln \ln k<\frac{k}{\ln k}<x
$$

and so

$$
\frac{1}{8} \ln x>\frac{1}{8} \ln \left(\ln \left(\frac{k+1}{x+1}\right)\right) .
$$

Step 2. Using the elementary inequality

$$
\ln (1+a) \geqslant a b \quad \text { for } \quad 0<b<1 \quad \text { and } \quad 0<a<\frac{1}{b}-1
$$

with $a=(k-x) /(x+1), b=1 /(2 \ln x)$, and

$$
0<a=\frac{k-x}{x+1} \leqslant \frac{k-x_{0}}{x_{0}+1}<\ln k-1<2 \ln x_{0}-1<2 \ln x-1=\frac{1}{b}-1 .
$$


we find that

$$
2 \ln x \ln \left(\frac{k+1}{x+1}\right)=2 \ln x \ln \left(1+\frac{k-x}{x+1}\right) \geqslant \frac{k-x}{x+1},
$$

and therefore,

$$
\frac{1}{4} \ln x \geqslant \frac{k-x}{8} \frac{1}{\ln \left(\frac{k+1}{x+1}\right)} \frac{1}{x+1}
$$

Step 3. Set

$$
f(x)=\frac{k-x}{8} \ln \left(\ln \left(\frac{k+1}{x+1}\right)\right)+\frac{1}{2} x \ln x .
$$

Clearly,

$$
f^{\prime}(x)=-\frac{1}{8} \ln \left(\ln \left(\frac{k+1}{x+1}\right)\right)-\frac{k-x}{8} \frac{1}{\ln \left(\frac{k+1}{x+1}\right)} \frac{1}{x+1}+\frac{1}{2}(\ln x+1) .
$$

Adding (A.10) and (A.11) we see $f^{\prime}(x) \geqslant 0$ and so

$$
\mathbf{I} \geqslant \exp \{f(x)\} \geqslant \exp \left\{f\left(x_{0}\right)\right\}
$$

Since $(k+1)\left(x_{0}+1\right)^{-1} \geqslant(3 / 4) \ln k$, we find

$$
\ln \left(\frac{k+1}{x_{0}+1}\right) \geqslant \ln \left(\frac{3 \ln k}{4}\right) \geqslant \frac{1}{2}(\ln \ln (k+2))^{\frac{1}{2}}, \quad k \geqslant 10,
$$

and so

$$
\begin{aligned}
f\left(x_{0}\right) & \geqslant \frac{1}{8}\left(k-x_{0}\right)\left(\frac{1}{2} \ln \ln \ln (k+2)-1\right)+\frac{1}{2} x_{0} \ln x_{0} \\
& =\frac{k}{16} \ln \ln \ln (k+2)+Z
\end{aligned}
$$

where

$\frac{2}{k} Z=-\frac{1}{8} \frac{\ln \ln \ln (k+2)}{\ln k}+\frac{1}{4 \ln k}+\frac{3}{4}-\frac{\ln \ln k}{\ln k} \geqslant \frac{3}{4}-\frac{9}{8} \frac{\ln \ln k}{\ln k} \geqslant 0, \quad k \geqslant 10$.

Combining (A.12) and (A.14) gives (A.9).

Case 2. Let $1<x \leqslant(k / \ln k)$. Using (A.13) we have

$$
\mathbf{I} \geqslant\left(\ln \left(\frac{k+1}{x+1}\right)\right)^{\frac{k-x}{2}} \geqslant\left(\frac{\ln \ln (k+2)}{2}\right)^{\frac{k}{4}\left(1-\frac{1}{\ln k}\right)} \geqslant 2^{-k}(\ln \ln (k+2))^{\frac{k}{8}} \text {. }
$$




\section{References}

[A] Artin, E.: Einführung in die Theorie der Gammafunktion. Hamburger Mathematische Einzelschriften 1. Verlag B. B. Teubner, Leipzig, 1931.

[Ber1] Bertoin, J.: Lévy Processes. Cambridge Tracts in Mathematics 121. Cambridge University Press. Cambridge, 1996.

[Ber2] Bertoin, J: Some properties of Burgers turbulence with white or stable noise initial data. In Levy processes, 267-279. Birkhäuser, Boston, 2001.

[DaPDe] Da Prato, G., Debussche, A.: Differentiability of the transition semigroup of the stochastic Burgers equations, and application to the corresponding Hamilton-Jacobi equation. Atti Accad. Naz. Lincei Cl. Sci. Fis. Mat. Natur. Rend. Lincei (9) Mat. Appl. 9 (1998), 267-277.

[JSh] Jacod, J., Shiryaev, A. N.: Limit Theorems for Stochastic Processes. Grundlehren Math. Wiss. 288. Springer-Verlag, Berlin, 1987.

[K1] Kolokoltsov, V. N.: Stochastic Hamilton-Jacobi-Bellman equation and stochastic Hamiltonian systems. J. Dynam. Control. Systems 2 (1996), no. 3, 299-319.

[K2] Kolokoltsov, V. N.: The stochastic HJB Equation and WKB Method. In Idempotency (Bristol, 1994), 285-302. Publ. Newton Inst. 11. Cambridge Univ. Press, Cambridge, 1998.

[K3] Kolokoltsov, V. N.: Semiclassical analysis for diffusions and stochastic processes. Lecture Notes in Math. 1724. Springer-Verlag, Berlin, 2000.

[KMa] Kolokoltsov, V. N., Maslov, V. P.: Idempotent analysis and its applications. Math. Appl. Kluwer, Dordrecht, 1997.

[KT1] Kolokoltsov, V. N., Tyukov. A. E.: The rate of escape of OrnsteinUhlenbeck process and the scattering theory for their perturbations. Markov Process. Related Fields 7 (2001), 603-625.

[KT2] Kolokoltsov, V.N., Tyukov. A.E.: Small time and semiclassical asymptotics for stochastic heat equations driven by a Lévy noise. Stoch. Stoch. Rep. 75 (2003), no. 1-2, 1-38.

[KwW] Kwapien, S., Woyczynski, W.: Random series and stochastic integrals: single and multiple. Probability and its Applications. Birkhäuser, Boston, MA, 1992.

[M] MÉtivier, M.: Semimartingales. A course on stochastic processes. De Gruyter Studies in Math. 2. Walter de Gruyter, Berlin-New York, 1982.

[Pro] Protter, P.: Stochastic integration and differential equations. A new approach. Applications of Mathematics 21. Springer-Verlag, Berlin, 1990.

[R] Rezakhanlou, F.: Central limit theorem for stochastic Hamilton-Jacobi equations. Comm. Math. Phys. 211 (2000), no. 2, 413-438.

[RoSa] Rosinski, J., Samorodnitsky, G.: Zero-one laws for multiple stochastic integrals. In Chaos expansions, multiple Wiener-Itô integrals and their applications (Guanajuato, 1992), 233-259. Probab. Stochastics Ser., CRC, Boca Raton, FL, 1994. 
[Sa] Sato, K.: Lévy processes and infinitely divisible distributions. Cambridge Studies Adv. Math. 68. Cambridge Univ. Press, Cambridge, 1999.

[So] Souganidis, P.: Stochastic homogenization of Hamilton-Jacobi equations and some applications. Asymptot. Anal. 20 (1999), no. 1, 1-11.

[Sz] Szulga, J.: Multiple stochastic integrals with respect to symmetric infinitely divisible random measures. Ann. Probab. 19 (1991), 1145-1156.

[Ta] TAKanobu, S.: Multiple stochastic integrals appearing in the stochastic Taylor expansions. J. Math. Soc. Japan 47 (1995), no. 1, 67-92.

[TrZ1] Truman, A., Zhao, H.: The stochastic Hamilton-Jacobi equation, stochastic heat equations and Schrödinger equations. In Stochastic Analysis and Applications (Powys, 1995), 441-464. World Sci. Publishing, River Edge, NJ, 1996.

[TrZ2] Truman, A., ZhaO, H.: Stochastic Hamilton-Jacobi equation and related topics. In Stochastic partial differential equations (Edinburgh, 1994), 287-303. London Math. Soc. Lecture Note Ser. 216. Cambridge Univ. Press, Cambridge, 1995.

Recibido: 25 de octubre de 2001

Revisado: 26 de julio de 2002

Vassili N.Kolokol'tsov
Department of Computing and Mathematics
Nottingham Trent University
Burton Street, Nottingham, NG14BU. United Kingdom
Vassili.Kolokoltsov@ntu.ac.uk
René L. Schilling
Department of Mathematics
University of Sussex
BN19RF. United Kingdom
r.schilling@sussex.ac.uk
Alexei E. Tyukov
School of Mathematics
Cardiff University
B.O Box 926, Cardiff CF244YH. United Kingdom
TyukovA@cardiff.ac.uk

Financial support for A. Tyukov through the NTU Research Enhancement Grant RF175 and for R. Schilling by Nuffield Foundation grant NAL/00056/G is gratefully acknowledged. We thank A. Truman, University of Wales (Swansea), for his support and valuable discussions. We are grateful to a very diligent referee who pointed out a serious measurability problem in an earlier version of this paper and whose critical comment helped us to improve the exposition in section 3 . 